\title{
A SUBGRID-SCALE MODEL FOR LARGE-EDDY SIMULATION OF PLANETARY BOUNDARY-LAYER FLOWS
}

\author{
PETER P. SULLIVAN, JAMES C. MCWILLIAMS, and CHIN-HOH MOENG \\ National Center for Atmospheric Research, ${ }^{*}$ Boulder, CO 80307, USA
}

(Received in final form 6 April, 1994)

\begin{abstract}
A long-standing problem in large-eddy simulations (LES) of the planetary boundary layer (PBL) is that the mean wind and temperature profiles differ from the Monin-Obukhov similarity forms in the surface layer. This shortcoming of LES has been attributed to poor grid resolution and inadequate sub-grid-scale (SGS) modeling. We study this deficiency in PBL LES solutions calculated over a range of shear and buoyancy forcing conditions. The discrepancy from similarity forms becomes larger with increasing shear and smaller buoyancy forcing, and persists even with substantial horizontal grid refinement. With strong buoyancy forcing, however, the error is negligible.

In order to achieve better agreement between LES and similarity forms in the surface layer, a two-part SGS eddy-viscosity model is proposed. The model preserves the usual SGS turbulent kinetic energy formulation for the SGS eddy viscosity, but it explicitly includes a contribution from the mean flow and a reduction of the contributions from the turbulent fluctuations near the surface. Solutions with the new model yield increased fluctuation amplitudes near the surface and better correspondence with similarity forms out to a distance of $0.1-0.2$ times the PBL depth, i.e., a typical surface-layer depth. These results are also found to be independent of grid anisotropy. The new model is simple to implement and computationally inexpensive.
\end{abstract}

\section{Introduction}

Large-eddy simulation (LES) is a well established tool for the study of turbulent flows (Wyngaard, 1984; Reynolds, 1989). The physical basis for LES is the separation of the flow into large-scale or resolved and small-scale or subgridscale (SGS) motions: the larger scales contain most of the energy and turbulent fluxes and are more dependent on the flow environment, while the less energetic SGS motions are believed to be more universal in character. The scale separation is determined by the choice of a spatial filter, which for practical purposes is fixed by the numerical resolution. Modeling the effects of the SGS motions on the resolved motions is a somewhat empirical science; however, it has been found that many aspects of LES solutions are relatively insensitive to the type of SGS model (Nieuwstadt et al., 1993), at least for conditions where the resolved motions are dominant.

Numerous SGS models have been proposed with varying levels of sophistication. In the context of planetary boundary-layer (PBL) flows, Deardorff (1980), Nieuwstadt and Brost (1986), and Moeng (1984) use an eddy-viscosity model based on a time-evolving turbulent kinetic energy equation; Mason and Callen

* The National Center for Atmospheric Research is sponsored by the National Science Foundation. 
(1986) use a variant of the Smagorinsky (1963) nonlinear-viscosity model; and Schmidt and Schumann (1989) use a second-order closure model. For channel flows driven by shear, recent SGS models have been developed by Horiuti (1993), Yakhot et al. (1989), Yoshizawa (1989), and Germano et al. (1991).

The variety of SGS models arises both because the theoretical justifications are arguable and because LES solutions are sensitive to the type of SGS model where the SGS motions are not conspicuously weaker than the resolved motions, e.g., near walls and in strongly stable density stratification. As evidence of this sensitivity, the turbulent motions in a LES of shear-driven channel flow cannot be sustained without reducing the dissipative effects of the SGS model (e.g., Moin and Kim, 1982). For shear flows, standard SGS models which use the theoretical values for the SGS constants are too dissipative with respect to the resolved motions. Recently Mason and Thomson (1992) showed that a typical LES wind-shear profile (i.e., the vertical derivative of the mean horizontal wind) deviates from the well established Monin-Obukhov similarity forms (Businger et al., 1971) near a bounding surface. They proposed a correction by introducing stochastic backscatter in their Smagorinsky SGS model that produced better agreement between LES and similarity forms; however, the computational cost of this model is high and the resulting resolved flow is significantly agitated by stochastic excitations, particularly near the boundary. Schumann (1993) pointed out that the addition of SGS stochastic backscatter significantly influences the resolved motions since a considerably larger eddy viscosity is required to dissipate the extra energy generated. Another known means for matching the similarity forms is by direct numerical simulation with finite viscosity (Spalart, 1988), but this conflicts with the LES goal of simulating fluid dynamics at large Reynolds number without the computational burden of resolving the viscous boundary layers.

There is increasing evidence that intermittent coherent structures carry most of the turbulent fluxes of momentum and buoyancy in the PBL and that these structures are preferentially generated near the ground (e.g., Mahrt and Gibson, 1992; Moeng and Sullivan, 1993). An important goal for LES is to represent at least the strongest of these structures. This, we believe, can be accomplished by allowing the resolved dynamics to be both weakly damped and weakly agitated by the SGS interactions, even in the vicinity of the boundary. Thus we accept the diagnosis of Mason and Thompson that current LES solutions are deficient in their surface layer by failing to match similarity forms for the first and second statistical moments of the turbulent PBL; however, our view is that both the statistical moments and the important dynamical motions can be recovered from the resolved motions by using a combination of a less dissipative SGS model and adequate grid resolution.

In this paper we examine in detail the surface layer of a PBL in LES solutions and the extent to which a new, simple SGS model is capable of diminishing their discrepancies from similarity forms. 


\section{Current SGS Model}

A common thread amongst the simpler SGS models is the assumption that the SGS stresses $\tau_{i j}$ can be approximately related to the resolved field by the adoption of a turbulent eddy viscosity $\nu_{t}$, so that

$$
\tau_{i j}=-2 \nu_{t} S_{i j}
$$

where the resolved flow rate of strain tensor is

$$
S_{i j}=\frac{1}{2}\left(\frac{\partial u_{i}}{\partial x_{j}}+\frac{\partial u_{j}}{\partial x_{i}}\right) .
$$

In Equation (2), $u_{i}$ are resolved velocity components $(u, v, w)$ in the coordinate directions $x_{i}$, i.e., $(x, y, z)$. SGS fluxes for buoyancy and/or scalar transport are assumed to be of the analogous form

$$
\tau_{\theta i}=-\nu_{\theta} \frac{\partial \theta}{\partial x_{i}}
$$

where $\nu_{\theta}$ is the eddy diffusivity for heat $\theta$ (or other scalar). The most widely used prescription for $\nu_{t}$ is the Smagorinsky (1963) nonlinear model, which results from an assumed local equilibrium balance between shear production and dissipation in the SGS turbulent kinetic energy (TKE) equation. For PBLs where buoyancy forcing is important, the Smagorinsky model is further modified (e.g., see Mason and Callen, 1986). An alternative SGS model is to solve the TKE equation explicitly, although such models have not been found to be generally superior to those obtained with a modified Smagorinsky model (Schmidt and Schumann, 1989). Advantages of such models are that no equilibrium assumption is required, and the prognostic equation provides a direct means of calculating the SGS kinetic energy which is needed to construct the actual pressure (see Equations (7) and (9) in Moeng, 1984).

For the sake of discussion and completeness, the SGS model based on the TKE equation developed by Deardorff (1980) and used by Moeng (1984), hereafter referred to as the baseline model, is briefly reviewed. This model uses Equations (1) and (3) with

$$
\nu_{t}=C_{k} l e^{1 / 2},
$$

and

$$
\nu_{\theta}=\left(1+\frac{2 l}{\Delta}\right) \nu_{t}
$$


where $e$ denotes the SGS kinetic energy, $l$ is a mixing length, $\Delta$ is an average grid mesh spacing, and $C_{k}$ is a constant. For the mixed spectral-finite difference code that we use,

$$
\Delta^{3}=\Delta x \Delta y \Delta z
$$

where $\Delta x, \Delta y$ and $\Delta z$ are effective grid mesh spacings, which account for de-aliasing of the upper $1 / 3$ of the wavenumbers. Here,

$$
\Delta x=3 L_{x} / 2 N_{x}, \Delta y=3 L_{y} / 2 N_{y}, \quad \text { and } \quad \Delta z=L_{z} / N_{z},
$$

where $\left(L_{x}, L_{y}, L_{z}\right)$ are the domain sizes and $\left(N_{x}, N_{y}, N_{z}\right)$ the number of grid points in the coordinate directions $(x, y, z)$, respectively. For unstable stratification, $l=\Delta$, while for stable stratification, $l$ is reduced as suggested by Deardorff $(1980)$,

$$
l=\frac{0.76 e^{1 / 2}}{\left(\frac{g}{\theta_{0}} \frac{\partial \theta}{\partial z}\right)^{1 / 2}},
$$

where $g$ is the gravitational acceleration and $\theta_{0}$ a reference virtual potential temperature. This length-scale correction for stable stratification was analyzed by Schumann (1991), and for the PBL cases studied had only minor effects. However, we found that this reduction in length scale is a necessary ingredient in order to model PBL flows with strong capping inversions. Recently we included a change in length scale near a bounding surface if the mixing length exceeds the value obtained from similarity theory, i.e., $\kappa z$, where $\kappa$ is the von Karman constant. The latter corresponds to a wall damping correction (Van Driest, 1956), but is rarely invoked even for the first grid point above the surface for grids which are not too anisotropic.

The prognostic equation for SGS energy $e$ is

$$
\left(\frac{\partial}{\partial t}+u_{j} \frac{\partial}{\partial x_{j}}\right) e=P+B-\epsilon+D
$$

where the various terms on the right-hand side are shear production $P$, buoyancy $B$, dissipation $\epsilon$, and diffusion $D$. Terms in the above expression can be directly computed or are modeled as

$$
\begin{aligned}
& P=-\tau_{i j} S_{i j}, \\
& B=\frac{g}{\theta_{0}} \tau_{\theta w}, \\
& \epsilon=C_{\epsilon} \frac{e^{3 / 2}}{l},
\end{aligned}
$$


and

$$
D=\frac{\partial}{\partial x_{i}}\left(2 \nu_{t} \frac{\partial e}{\partial x_{i}}\right) .
$$

If the LES grid is within the inertial subrange, the spectral analysis of Moeng and Wyngaard (1988) finds that the SGS constants are $C_{k}=0.1$ and $C_{\epsilon}=$ 0.93 . Their computations of a highly convective PBL are found to be relatively insensitive to modest changes in these constants. If the shear production and dissipation terms are equated in Equation (8) and Equations (1) and (4) are used, the Smagorinsky model

$$
\nu_{t}=\left(C_{s} \Delta\right)^{2} \sqrt{2 S_{i j} S_{i j}},
$$

is recovered where the Smagorinsky constant $C_{s}$ written in terms of $C_{k}$ and $C_{\epsilon}$ is

$$
C_{s}=\left(C_{k} \sqrt{\frac{C_{k}}{C_{\epsilon}}}\right)^{1 / 2} .
$$

Substitution of the theoretical values noted previously for $C_{k}$ and $C_{\epsilon}$, yields $C_{s}=0.18$.

Additional ingredients of the baseline SGS model are the surface boundary conditions. We follow the approach developed by Moeng (1984) and others in which horizontally-averaged conditions at the first computational grid point above the surface are matched to Monin-Obukhov similarity theory (Businger et al., 1971). This assumes that the vertical grid is adequate to resolve at least a portion of the surface layer. The outcome of this procedure is a prediction of the horizontally-averaged value of the surface shear stress, and the surface potential temperature or heat flux depending on the form of the boundary condition for the latter. In order to estimate fluctuating values of the surface stress, a "local similarity rule" is adopted which relates the fluctuating values of the surface stress and temperature to the fluctuating velocity and temperature at the first grid point. Numerous rules have been proposed for this step, e.g., Schumann (1975), Mason and Callen (1986), and Piomelli et al. (1989), but we have found that all the suggested schemes alter the computed solutions only slightly. Thus in the present work we adopt the scheme suggested in Moeng (1984).

\section{Proposed SGS Model}

As a bounding surface is approached, the mean shear increases, anisotropy is induced in the turbulent velocity components, and the dominant scales in the turbulent motion decrease in size. These factors, especially the latter, erode the ability to perform accurate LES calculations near rigid boundaries because a greater 
burden is placed on the form of the SGS model. As a boundary is approached, a transition is needed from the LES solution, where the majority of turbulent motions are computed, towards ensemble-average modeling, where all the turbulent motions are subgrid scale. This transition near a boundary is necessitated because the LES grid is fixed and the scales of the important turbulent motions are decreasing. The ensemble-average model equations, which are a greatly simplified subset of the LES equations, are known to reproduce mean logarithmic profiles when the turbulence parameterization is an eddy viscosity that varies linearly with distance from the wall (e.g., Cebecci and Bradshaw, 1988). However, in the ensemble-average equations, the turbulence parameterization differs from standard SGS models in that it encompasses all turbulent scales. Thus it is possible to envision a transition from LES to ensemble-average modeling in the vicinity of the wall in the framework of the LES equations, provided the SGS model is appropriately modified.

Most SGS models are developed by first assuming that the flow contains an inertial subrange so that the SGS motions can be considered to be isotropic. Then the SGS constants are computed by adopting a specific form for the isotropic energy spectrum (Moeng and Wyngaard, 1988). The isotropy assumption is satisfied provided that the grid resolution is extremely fine so that the anisotropy introduced by the mean shear is negligible (Schumann, 1975). However, for a high Reynolds number large-eddy simulation of a PBL (i.e., where the molecular viscosity is not explicitly accounted for), somewhere near the bounding surface the effect of the mean shear on the SGS motions can no longer be ignored, no matter what the grid resolution. Consequently, the SGS modeling that is appropriate for mid-PBL must be altered to account for the special conditions near a boundary. Further, the optimum value of the SGS constants can be expected to deviate from the theoretical predictions in the interior of the flow.

In view of the above arguments, the baseline model needs to be extended to account for the inhomogeneous nature of the flow in the $z$ direction, and the limited resolution of an LES near a bounding surface. At the same time, it is desirable that the baseline model is recovered in the regions away from a bounding surface. This is advantageous in view of the success of the well established baseline model.

Near a bounding surface, PBL flows take on the character of a shear-driven channel flow, and thus it is natural to adopt SGS modeling ideas used in the LES of shear-dominated flows. A potential starting point for a SGS model is the two-part eddy viscosity model first proposed by Schumann (1975), extended by Grotzbach and Schumann (1977) and Schumann et al. (1980), and further employed by Moin and Kim (1982). In these studies, the computation of the SGS stresses is split into what are termed isotropic and inhomogeneous contributions. The isotropic eddy viscosity depends on the magnitude of the fluctuating strain rate, while the inhomogeneous term varies with the magnitude of the mean strain rate. For a shear-driven boundary-layer flow, the inhomogeneous term is only 
significant near the wall where $\partial\langle u\rangle / \partial z$ is large. Satisfactory predictions are obtained with the two-part model for channel flows, but only with a Smagorinsky constant smaller than the theoretical estimate based on the assumption of an isotropic spectrum in the inertial range. An important feature of the two-part eddy viscosity model, owing to its separation into mean and fluctuating parts, is that it provides a transition from LES to ensemble-average modeling as the wall is approached. In the two-part model as used by Schumann (1975), the fluctuating eddy viscosity decreases roughly like the resolved fluctuating strain in the near-wall region.

Our proposed SGS model which embodies the above ideas is

$$
\tau_{i j}=-2 \nu_{t} \gamma S_{i j}-2 \nu_{T}\left\langle S_{i j}\right\rangle
$$

Here the angle brackets \langle\rangle denote averages over the homogeneous directions, in this case $x$ and $y$, and $\nu_{T}$ is an average eddy viscosity which needs to be expressed in terms of mean flow quantities. Hereafter $\nu_{t}$ and $\nu_{T}$ are referred to as fuctuating and mean-field eddy-viscosities, respectively. In Equation (15), $\gamma$, referred to as an "isotropy factor", has a dual interpretation in that it accounts for variability in the theoretical SGS constants due to anisotropy of the mean flow, but also controls the transition between SGS and ensemble-average turbulence parameterizations. The exact forms of $\nu_{T}$ and $\gamma$ are given in Sections 3.1 and 3.2 , respectively.

The fluctuating eddy viscosity, $\nu_{t}$, in Equation (15) is taken to be the baseline model given by Equation (4) with a noticeable exception in the computation of the SGS energy, e. Here, the shear production term in the SGS energy Equation (8) is computed solely from the fiuctuating velocities as suggested by Schumann (1975). In other words, the mean-shear effect is taken out from the production term in Equation (8) so that

$$
P=2 \nu_{t} \gamma\left(S_{i j}-\left\langle S_{i j}\right\rangle\right)\left(S_{i j}-\left\langle S_{i j}\right\rangle\right)
$$

We realize that the exact forms of Equations (15) and (16) are not unique, and in fact other functional forms are possible. However, these two expressions do satisfy what we view as an important requirement, i.e., the turbulence parameterizations pass smoothly from a standard SGS model to one based on ensembleaverage closure modeling depending on the level of the resolved fluctuating strain compared to the mean strain. As noted by Schumann (1975), the use of fluctuating strains in the production term is consistent with the assumption of local isotropy and moreover results in zero production of SGS energy if the resolved turbulence disappears. Under the assumption made in Equation (16), the mean shear can only directly generate resolved-scale turbulence, but not SGS motions. Of course, the mean shear effect still influences the SGS motions via the resolved-field strain rate. 


\subsection{MFAN-FIELD EDDY-VISCOSITY MODEL}

The specification for $\nu_{T}$ is devised to recover law-of-the-wall behavior in the absence of any resolved turbulence. A model which is consistent with this idea is Prandtl's mixing length concept, viz.,

$$
\nu_{T}=\left(C_{K} L_{m}\right)^{2} \sqrt{2\left\langle S_{i j}\right\rangle\left\langle S_{i j}\right\rangle} .
$$

With the definition for $S_{i j}$ (Equation (2)), Equation (17) simplifies to

$$
\nu_{T}=\left(C_{K} L_{m}\right)^{2} \sqrt{\left(\frac{\partial\langle u\rangle}{\partial z}\right)^{2}+\left(\frac{\partial\langle v\rangle}{\partial z}\right)^{2}}
$$

for horizontally homogeneous PBL flows. In the above expressions, $C_{K}$ is a constant and $C_{K} L_{m}$ is a mixing-length scale tied to the grid mesh spacing. Schumann (1975), Grotzbach and Schumann (1977), and Schumann et al. (1980) find that the constant analogous to $C_{K}$ is approximately 0.1 for channel flows. This constant is, however, influenced by the grid geometry and a universal value is difficult to prescribe. Despite the attempts by Schumann (1975) and Grotzbach and Schumann (1977) to derive theoretical estimates for these constants, they were forced to introduce additional corrective constants to gain agreement over a range of experiments.

Our choice of $C_{K}$ is instead guided by the desire to match Monin-Obukhov similarity theory in the wall region. The baseline model calculations already make use of similarity theory by invoking a match between the computed mean wind and Monin-Obukhov similarity predictions at the first $u$ grid point $(\Delta z / 2)$ and thereby determine the surface friction velocity (Moeng, 1984). (Recall that the $u$ and $w$ grid levels are staggered so that the first $u$ grid location is at $\Delta z / 2$.) With the introduction of the mean-field eddy-viscosity term in Equation (15), an additional constraint can be imposed on our matching procedure. Here we stipulate that the computed and similarity-theory derivatives also match at the first $w$ grid point, $z=z_{1}=\Delta z$. The expression for the wind-speed derivative (e.g., Businger et al., 1971) is simply

$$
\frac{\partial U_{s}}{\partial z}=\frac{u_{*} \phi_{m}}{\kappa z}
$$

where $u_{*}$ is the friction velocity, $\kappa$ the von Karman constant, $\phi_{m}$ the MoninObukhov stability function for momentum, and $U_{s}$ the average surface layer wind speed (i.e., $U_{s}=\left\langle\sqrt{u^{2}+v^{2}}\right\rangle$ ). In order to make use of Equation (18), we further impose a "constant flux" condition below $z=z_{1}$. For our LES calculations, this condition states that the sum of the SGS and resolved momentum fluxes be equal to the surface stress, i.e.,

$$
\left[\left\langle\tau_{u w}\right\rangle^{2}+\left\langle\tau_{v w}\right\rangle^{2}\right]^{1 / 2}+\left[\langle u w\rangle^{2}+\langle v w\rangle^{2}\right]^{1 / 2}=u_{*}^{2} .
$$


We develop a simple predictive relationship for the mean-field eddy-viscosity at the first grid point $z_{1}, \nu_{T}^{*}=\nu_{T}\left(z_{1}\right)$, by invoking two approximations. First, we neglect the fluctuating components of strain compared to the mean strain so that only the horizontally-averaged SGS stress in Equation (15) is retained. This leads to

$$
\begin{aligned}
& \left\langle\tau_{u w}\right\rangle=-\left(\left\langle\nu_{t} \gamma\right\rangle+\nu_{T}\right) \frac{\partial\langle u\rangle}{\partial z} \\
& \left\langle\tau_{v w}\right\rangle=-\left(\left\langle\nu_{t} \gamma\right\rangle+\nu_{T}\right) \frac{\partial\langle v\rangle}{\partial z}
\end{aligned}
$$

and, secondly, we ignore the mean wind turning with height at the first grid point which implies that

$$
\sqrt{\left(\frac{\partial\langle u\rangle}{\partial z}\right)^{2}+\left(\frac{\partial\langle v\rangle}{\partial z}\right)^{2}} \approx \frac{\partial U_{s}}{\partial z}
$$

Upon substitution of Equation (21) into Equation (20) and making use of Equations (19) and (22), the expression for the mean-field eddy-viscosity, $\nu_{T}^{*}$, becomes

$$
\nu_{T}^{*}=\frac{u_{*} \kappa z_{1}}{\phi_{m}\left(z_{1}\right)}-\left\langle\nu_{t} \gamma\right\rangle-\frac{\kappa z_{1}}{u_{*} \phi_{m}\left(z_{1}\right)}\left[\langle u w\rangle^{2}+\langle v w\rangle^{2}\right]^{1 / 2} .
$$

Equation (23) provides an adaptive method for estimating the mean-field eddy viscosity needed to force the computed wind speed derivative to match with similarity theory at $z=z_{1}$. At any other height, we use

$$
\nu_{T}=\nu_{T}^{*} \frac{\kappa z_{1}}{u_{*} \phi_{m}\left(z_{1}\right)} \sqrt{2\left\langle S_{i j}\right\rangle\left\langle S_{i j}\right\rangle}
$$

which follows directly from Equations (17), (19), and (22).

For consistency, a similar correction should also be applied to the SGS temperature (or scalar) field, and several variants analogous to Equation (15) were tried for $\tau_{\theta i}$. However, we found that correcting the velocity field induces a substantial improvement in the mean potential-temperature profile; hence no additional correction is applied to Equation (3) for the heat flux.

\subsection{ISOTROPY FACTOR}

It is well known, from trial and error, that the optimum value of the Smagorinsky constant decreases with increasing mean shear. For instance, $C_{s}=0.2$ in homogeneous isotropic flows (Clark et al., 1979), while in channel flows, Deardorff (1970) and Piomelli et al. (1988) use $C_{s}=0.1$; Bardina (1983) finds an optimum value of $C_{s}=0.09$, and Moin and $\mathrm{Kim}$ (1982) use $C_{s}=0.065$. Since the equivalent Smagorinsky constant based on the inertial subrange spectrum is 
$C_{s}=0.18$, we can expect that this value of $C_{s}$ is too large (i.e., too dissipative) for use near a solid surface.

The exact cause for the observed dependence of $C_{s}$ on mean shear is not completely understood, but Horiuti (1993) and Yakhot et al. (1989) speculate that the anisotropy of the SGS motions is significant near a bounding surface. In general, a decrease in the Smagorinsky constant coincides with an increase in anisotropy in both the resolved and SGS velocities (Horiuti, 1993). It should be noted that the change in the Smagorinsky constant considered here is a consequence of the mean shear. We realize that the Smagorinsky constant also varies with grid mesh aspect ratio as pointed out by Scotti et al. (1993).

In order to account for this anisotropic effect, Yakhot et al. (1989) devised a correction based on the ratio of the resolved normal velocity component to the total resolved energy, i.e., $3 w^{2} /\left(u^{2}+v^{2}+w^{2}\right)$, which is applied on an instantaneous basis. Yakhot's proposal makes an attempt to address the anisotropy issue, but as noted by Horiuti (1993), this correction does not account for a change in isotropy of the SGS motions with increasing grid resolution. Horiuti (1993) proposed a similar correction as Yakhot et al. (1989) with filtered velocity components. Then in the limit of very fine grid resolution, the isotropy correction tends to unity. The effect of the Yakhot et al. (1989) and Horiuti (1993) isotropy correction is to act much like a wall damping function.

Both of these models neglect the source of the anisotropy for the smallscale motions, which is the presence of increasing mean shear near the wall. As pointed out by Tennekes and Lumley (1972), the small-scale motions will be roughly independent of the mean strain rate, and hence isotropic, if the ratio of the small-scale to large-scale strain is larger than about 10 . Also this ratio is dependent on grid resolution, since the small-scale (high wavenumber) strain rate varies like $\Delta^{-2 / 3}$ in the inertial subrange. Consequently, we choose to base an isotropy factor on the ratio of small- and large-scale strain rates in view of their important physical content and their ready availability in an LES. Here the horizontally-averaged fluctuating resolved strain,

$$
S^{\prime}=\sqrt{2\left\langle\left(S_{i j}-\left\langle S_{i j}\right\rangle\right)\left(S_{i j}-\left\langle S_{i j}\right\rangle\right)\right\rangle},
$$

is used as an estimator for the small-scale strain, while the large-scale strain is simply determined from the mean strain

$$
\langle S\rangle=\sqrt{2\left\langle S_{i j}\right\rangle\left\langle S_{i j}\right\rangle} .
$$

Then the isotropy factor is defined as

$$
\gamma=\frac{S^{\prime}}{S^{\prime}+\langle S\rangle} \text {. }
$$

Notice that Equation (27) is solely a function of $z$ since the horizontal average of the fluctuating strain rate is used in Equation (25). For fixed $S^{\prime}$, the 
asymptotic limits of Equation (27) are unity (zero) when the mean strain $\langle S\rangle$ is small (large). Very near the wall where mean strain dominates the resolved strain, $S \sim 1 / \kappa z$ and $\gamma \sim S^{\prime} \kappa z$. Also, if we use Equation (13) for $\nu_{t}$, then the equivalent Smagorinsky model is

$$
\nu_{t} \gamma=\left(\hat{C}_{s} \Delta\right)^{2} \sqrt{2 S_{i j} S_{i j}}
$$

where the equivalent Smagorinsky constant $\hat{C}_{s}$ is

$$
\hat{C}_{s}=C_{s} \sqrt{\gamma}
$$

As alluded to earlier, $\gamma$ can be interpreted as an isotropy measure, but this also facilitates a transition or blending of SGS and ensemble-average turbulence parameterizations. Since $\gamma$ decreases as the ratio of resolved fluctuating strain to mean strain, it reduces the net eddy viscosity $\nu_{t} \gamma$ and thereby leads to increasing importance of the mean-field viscosity.

\subsection{Matching with the Baseline model}

The mean-field eddy viscosity $\nu_{T}$ and isotropy factor $\gamma$ play important roles near a bounding surface where mean shear is large. Away from the wall in the mid$\mathrm{PBL}$, the fluctuating resolved strain is almost a factor of 10 larger than the mean strain for typical grid resolutions used (see Section 4). In this region, $\gamma \rightarrow 1$ and $\nu_{T} \ll \nu_{t}$. Therefore for $z>z_{i} / 2$, where $z_{i}$ is the depth of the PBL, we choose to set $\gamma=1$ and $\nu_{T}=0$. The eddy-viscosity model given by Equation (15) then reverts to the baseline model in the upper PBL. Then, the baseline model is used in regions of strong stable stratification that occur near $z=z_{i}$, where the form of $\nu_{T}$ and $\gamma$ are not expected to apply. The combined effects of shear and significant stable stratification are an active area of investigation (e.g., Gerz and Schumann, 1989; and Canuto and Minotti, 1993), and will not be considered here. We realize that the selection of $0.5 z_{i}$ as the matching height is arbitrary, and any choice in the range $0.3 z_{i}<z<0.7 z_{i}$ would suffice. The intent is to use the new model in the surface layer, but not in the entrainment layer.

\subsection{Features of the proposed Model}

The proposed SGS model borrows ideas advanced by other investigators but differs in several aspects. First, we prefer to keep the mean shear in the first term on the right-hand side of Equation (15), whereas previous proposals eliminate the mean shear from this term. As a result, the new model for the SGS stresses given by Equation (15) can blend smoothly with the baseline model given by Equation (1) since the mean field eddy viscosity becomes negligibly small away from the surface layer. Secondly, Moin and Kim (1982) choose the length scale associated with the mean-field eddy viscosity $\nu_{T}$ to be dependent only on the spanwise grid spacing. This selection was based on the observation that the 
spanwise spacing of the streaks was the dominant motion that was inadequately resolved. Our procedure for determining the mean-field surface eddy viscosity, $z_{T}^{*}$, avoids a direct specification of the mixing length $C_{K} L_{m}$ as is required in Schumann (1975). This is important because the wall streaks in our PBL flows with Coriolis rotation are oriented with the surface wind (Moeng and Sullivan, 1994) and not solely in the $x$ direction as in channel flow.

Another advantage of the present scheme is that it matches the velocity derivative near the surface to the similarity relation independent of grid resolution. In Equation (15), the mean-field eddy viscosity is introduced to account for inadequate grid resolution and for shortcomings in the baseline SGS model. This term dies out quickly away from the wall so that the baseline model is recovered. Also noteworthy is that $\nu_{T}\left\langle S_{i j}\right\rangle$ in Equation (15) is only a function of $z$ and, as mentioned by Moin and Kim (1982) and Rogallo and Moin (1984), only dissipates energy associated with the mean flow $U_{s}$. This directly influences the mean flow profile and indirectly leads to more resolved flow turbulence through the energy cascade.

\section{Results}

A sequence of large-eddy simulations with varying grid densities in different flow regimes was carried out using the baseline and new SGS models. The PBL flows vary from strongly convective to shear-dominated (or neutral). A strong stably stratified capping inversion was used to limit the growth of the PBL. A gridresolution sensitivity study was further carried out for a weakly convective PBL with vigorous shear forcing. A summary of various properties of the simulations and global statistics is provided in Tables I and II. The simulations are labeled as strongly convective (B), or as shear-dominated with either zero $(S)$ or weakly convective $(S B)$ buoyancy forcing. Mean and turbulent statistics are gathered over about two large-eddy turnover times after statistically steady conditions were reached. More information concerning these simulations can be found in Moeng and Sullivan (1994).

\subsection{SGS EDDY-VISCOSITY PROFILES}

To see how the resolved flow is sensitive to the type of SGS model very near the surface, we examine the eddy-viscosity profiles for the baseline and new models. In Figure 1, the horizontally-averaged eddy-viscosity profiles $\nu_{t}$, for the baseline model, and $\nu_{t} \gamma$ and $\nu_{T}$, for the new model, are shown for each of the three basic flows considered. These simulations differ slightly in numbers of grid points used (see Table I), but are roughly comparable in terms of grid resolution. In the case of the new SGS model, the product of the fluctuating eddy viscosity and the isotropy factor is shown since this is the effective SGS diffusivity seen by the resolved flow. 
TABLE I

Input parameters for simulations

\begin{tabular}{llrcll}
\hline Case & $\begin{array}{l}L_{x}, L_{y} \\
(\mathrm{~m})\end{array}$ & $\begin{array}{l}L_{z} \\
(\mathrm{~m})\end{array}$ & $N_{x} x N_{y} x N_{z}$ & $\begin{array}{l}Q_{*} \\
\left({ }^{\circ} \mathrm{K} \mathrm{m} / \mathrm{s}\right)\end{array}$ & $\begin{array}{l}U_{g} \\
(\mathrm{~m} / \mathrm{s})\end{array}$ \\
\hline$S$ & 3000 & 1000 & $96 \times 96 \times 96$ & 0.0 & 15 \\
$S B 1$ & 2000 & 750 & $50 \times 50 \times 75$ & 0.02 & 15 \\
$S B 2$ & 2000 & 750 & $64 \times 64 \times 75$ & 0.02 & 15 \\
$S B 3$ & 2000 & 750 & $100 \times 100 \times 75$ & 0.02 & 15 \\
$S B 4$ & 2000 & 750 & $192 \times 192 \times 75$ & 0.02 & 15 \\
$B$ & 5000 & 2000 & $96 \times 96 \times 96$ & 0.24 & 10 \\
\hline
\end{tabular}

TABLE II

Global statistics from simulations. The PBL depth $z_{i}$ is defined as the vertical location where the buoyancy flux is minimum and $\tau_{*}$ is the large eddy turnover time; $\tau_{*}=z_{i} / w_{*}\left(\right.$ or $z_{i} / u_{*}$ ) for convective (or neutral) flows.

\begin{tabular}{lllrll}
\hline Case & $\begin{array}{l}u_{*} \\
(\mathrm{~m} / \mathrm{s})\end{array}$ & $\begin{array}{l}w_{*} \\
(\mathrm{~m} / \mathrm{s})\end{array}$ & \multicolumn{1}{l}{$z_{i}$} & \multicolumn{1}{c}{$\tau_{*}$} & $-z_{i} / L$ \\
& $(\mathrm{~s})$ & \\
\hline$S^{b}$ & 0.499 & 0.0 & 466 & 934 & 0.0 \\
$S B 1^{b}$ & 0.596 & 0.682 & 484 & 711 & 0.596 \\
$S B 2^{b}$ & 0.581 & 0.680 & 482 & 710 & 0.641 \\
$S B 3^{b}$ & 0.584 & 0.684 & 492 & 718 & 0.645 \\
$S B 4^{b}$ & 0.573 & 0.683 & 489 & 715 & 0.678 \\
$B^{b}$ & 0.556 & 2.000 & 1042 & 520 & 18.9 \\
$S^{n}$ & 0.536 & 0.0 & 532 & 993 & 0.0 \\
$S B 1^{n}$ & 0.620 & 0.683 & 488 & 714 & 0.534 \\
$S B 2^{n}$ & 0.613 & 0.680 & 481 & 707 & 0.545 \\
$S B 3^{n}$ & 0.617 & 0.685 & 491 & 717 & 0.547 \\
$S B 4^{n}$ & 0.629 & 0.684 & 490 & 716 & 0.512 \\
$B^{n}$ & 0.570 & 2.000 & 1028 & 514 & 17.5 \\
\hline
\end{tabular}

${ }^{b}$ Simulation with baseline SGS model.

${ }^{n}$ Simulation with new SGS model.

Features worth noting in Figure 1 are that the baseline and new models yield nearly identical eddy viscosities for all three flows above $z / z_{i}>0.2$, suggesting that the character of the baseline solutions is preserved in the new calculations in this region. Below $z / z_{i}>0.2$, the fluctuating eddy viscosity $\nu_{t} \gamma$ in the new model is smaller than its baseline model counterpart. At the first $w$ grid point, $\nu_{t} \gamma$ 


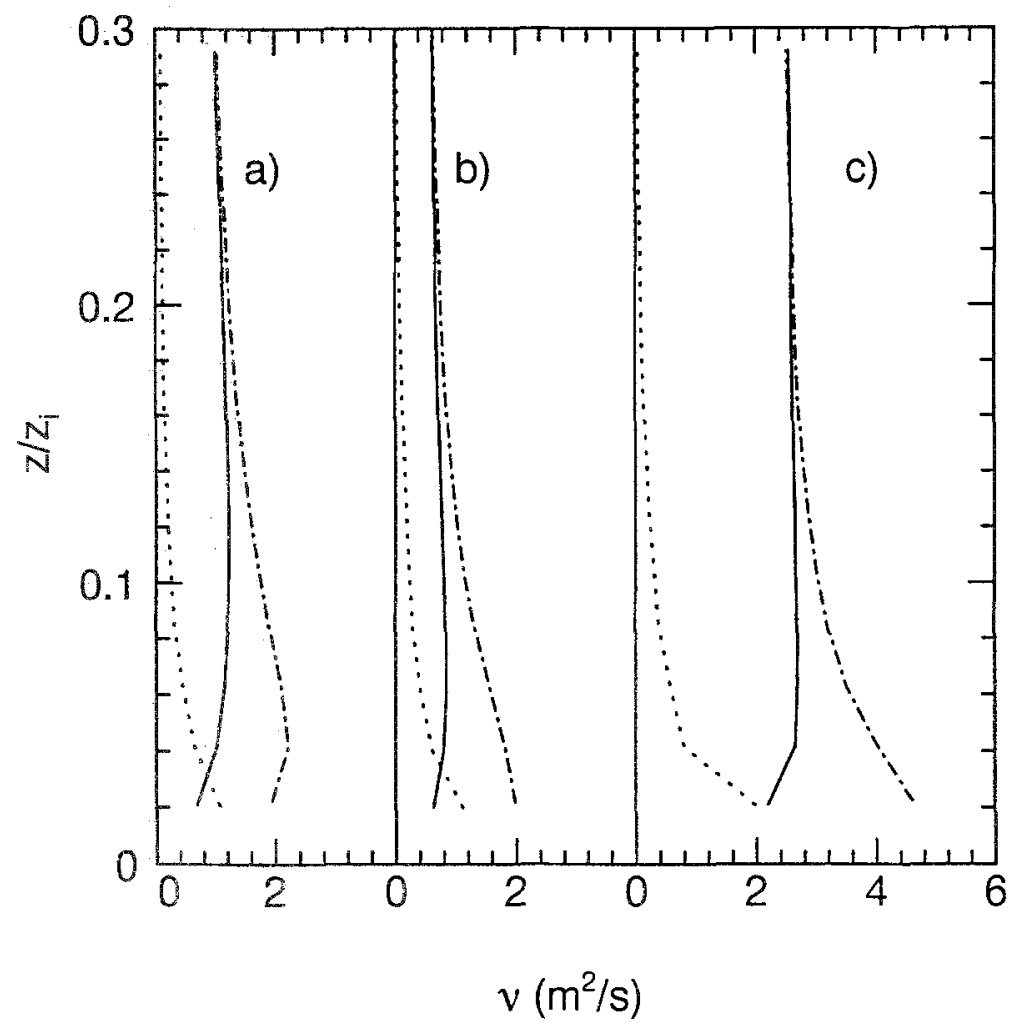

Fig. 1. SGS eddy viscosity profiles dashed-dotted line $\nu_{t}$ baseline model, solid line $\nu_{t} \gamma$, dotted line $\nu_{T}$ new model; (a) neutral flow simulation $S$, (b) slight convective-strong shear flow simulation $S B 3$, (c) strong convective flow simulation $B$.

is more than $50 \%$ smaller than $\nu_{t}$ for an three flows. This is a direct consequence of the increasing dominance of the mean strain $\langle S\rangle$ compared to the resolved fluctuating strain $S^{\prime \prime} \cdot \gamma$ becomes smaller as the surface is approached where $\langle S\rangle>S^{\prime}$. It is interesting to observe that the mean-field eddy viscosity $\nu_{T}$ is smaller than its fluctuating counterpart over the entire domain except for a region close to the wall for simulations $S$ and $S B 3$. In the strongly convective simulation $B$, the fluctuating and mean-field eddy viscosities at the first grid level are about the same magnitude. These results also suggest that the assumption that $\nu_{T}$ is negligible compared to $\nu_{t}$, discussed in Section 3.3, is certainly justified above $z / z_{i}>0.2$. The lower value of the SGS fluctuating eddy viscosity, and hence dissipation, in the new model implies the resolved motions are more energetic in the surface layer compared to results obtained with the baseline model. At the same time, the mean-field eddy viscosity helps to improve the shape of the mean velocity and temperature profiles near the boundary, as shown below. 


\subsection{COMPARISON WITH SIMILARITY THEORY PREDICTIONS}

In view of our focus on the surface layer, the present simulations are compared in detail with the predictions from similarity theory. In other words, similarity theory in the surface layer is considered the standard, and small or large deviations from it are accordingly judged as either good or bad. Comparison quantities are the stability functions for momentum and heat, and the wind and temperature profiles. For the strong convective PBL flow studied, where $-z_{i} / L>10$, similarity theory is expected to be applicable up to about $0.1 z_{i}$ (Wyngaard, 1988). The depth of the surface layer is expected to extend beyond $0.1 z_{i}$ for the other simulations since $-z_{i} / L<1$.

In the surface layer, the logarithmic velocity and temperature profiles found in neutral boundary-layer flows are modified by stratification effects. The derivatives of the mean wind speed $U_{s}$ and mean potential temperature $\Theta$ are defined as

$$
\phi_{m}=\frac{\kappa z}{u_{*}} \frac{\partial U_{s}}{\partial z}
$$

and

$$
\phi_{s}=\frac{\kappa z}{\theta_{*}} \frac{\partial \Theta}{\partial z}
$$

where $\phi_{m}$ and $\phi_{s}$ are so-called stability functions for momentum and heat, respectively. The surface heat flux $Q_{*}$, and friction velocity $u_{*}$ are used to define $\theta_{*}$, i.e., $\theta_{*}=-Q_{*} / u_{*}$. On the basis of experimental results and dimensional analysis, Businger et al. (1971) find for unstable and neutral conditions that

$$
\phi_{m}=(1-15 \zeta)^{-1 / 4} \text {, }
$$

and

$$
\phi_{s}=0.74(1-9 \zeta)^{-1 / 2},
$$

where $\zeta=-z / L$. In the case of zero surface heat flux (i.e., the neutral case) $\phi_{m}=1$ for all $z$. Note that Equations (30) and (31) can be integrated for the wind speed and potential temperature. (These expressions are not given here, but are supplied in Businger et al. (1971). There is some debate about the exact functional form for the stability functions, but the above expressions suffice for the present discussion. We are aware that the roughness length $z_{0}$ should be included in the expressions for the stability functions; however, for the grids used, $z_{1} \gg z_{0}$, and the effects of $z_{0}$ can be neglected. As pointed out by Businger et al. (1971), the effect of $z_{0}$ cannot, however, be neglected in the integrated expressions for the wind speed and potential temperature.

The stability functions are dimensionless forms of the vertical wind speed and potential temperature derivatives, and can be used to assess the agreement 


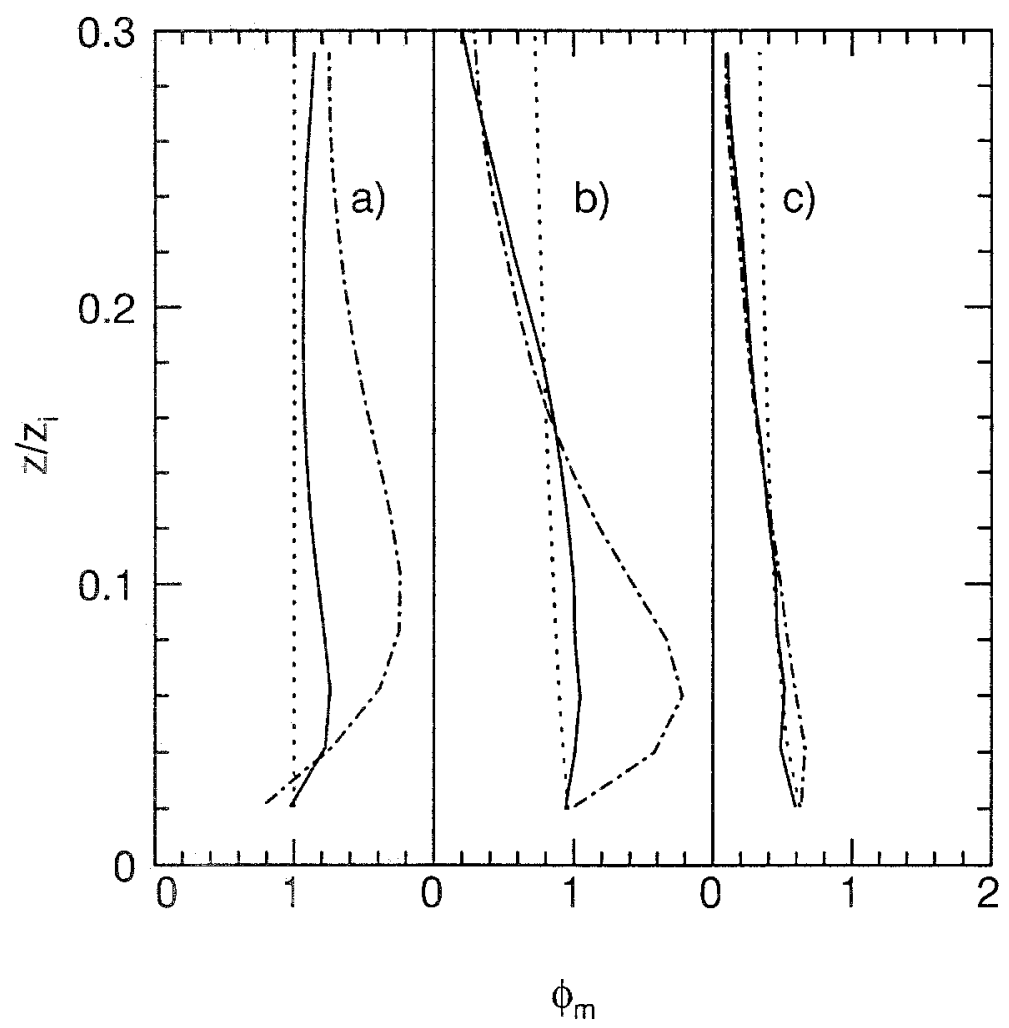

Fig. 2. Momentum stability function profiles; dotted line similarity theory, dashed-dot line baseline model, solid line new model; for the same flows as in Figure 1.

between LES and similarity theory predictions. LES estimates for the stability functions are obtained by substitution of horizontal- and time-averaged profiles of wind speed and potential temperature into the right-hand side of Equations (30) and (31). These same averaged wind speed and potential temperature LES fields are also compared to similarity-theory predictions for $U_{s}$ and $\Theta$.

A comparison of LES $\phi_{m}, U_{s}, \phi_{s}$ and $\Theta$ with their similarity-theory counterparts for the three flows $S, S B 3$ and $B$ are shown in Figures 2, 3, 4 and 5, respectively. Here the wind profiles are shown in terms of the dimensionless coordinates $U_{\mathcal{s}} / u_{*}$ and $z / z_{0}$, where the roughness length $z_{0}=0.16 \mathrm{~m}$. The potential temperature profiles are also displayed in the dimensionless form $\left(\theta-\theta_{s}\right) / \theta_{*}$, where $\theta_{s}$ is the surface temperature.

The stability function for the baseline model is in close agreement with the Mason and Thomson (1992) result for a neutrally stratified Ekman flow without backscatter. Almost a factor of two overshoot in the computed $\phi_{m}$ occurs near $z / z_{i}=0.1$. This implies that our neutral PBL with a strong capping inversion exhibits the same "error" as observed by Mason and Thomson. The agreement between the computed $\phi_{m}$ and its similarity counterpart is noticeably improved 


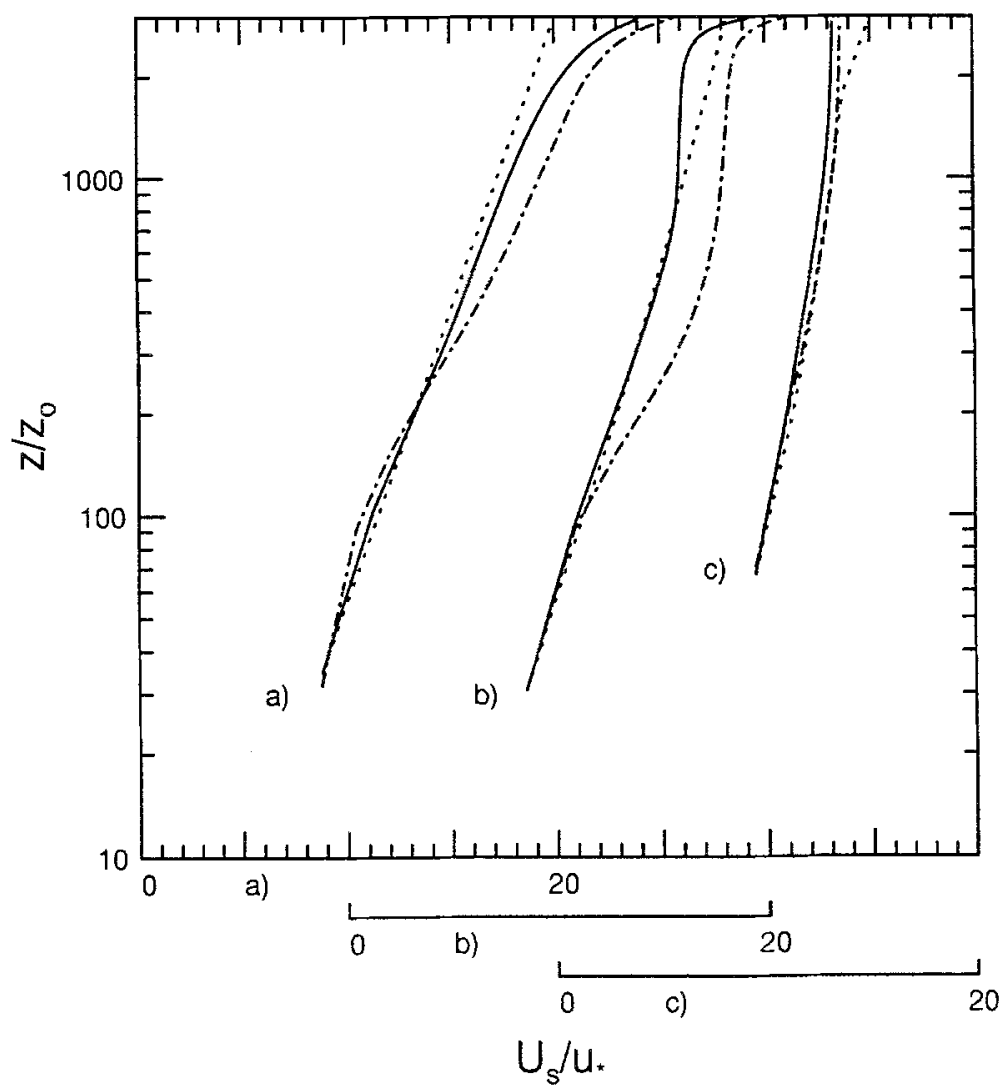

Fig. 3. Wind speed profiles; dotted line similarity theory, dashed-dotted line baseline model, solid line new model; for same flows as in Figure 1.

using the new SGS model for the neutral and weakly convective flows. The strong convective flow, $B$, apparently is relatively insensitive to the SGS model. These results indicate that the excessive mean-velocity gradients obtained using the baseline model are reduced considerably using the new model for simulations $S$ and $S B 3$.

The computed LES wind profiles, shown in Figure 3, show marked improvement with the new model, reflecting the enhanced agreement in the stability function. Clear logarithmic velocity profiles are observed for all three simulations starting from the first grid point and extending up to about $z / z_{0}=800$ (or about $z \approx 128 \mathrm{~m}$ ) with the new SGS model. The simulations with the baseline model deviate sharply from similarity theory except very close to the wall. It should be noted that the effect of "stochastic backscatter" (Mason and Thomson, 1992) has the same qualitative improvement as the findings obtained here with the new SGS model. 


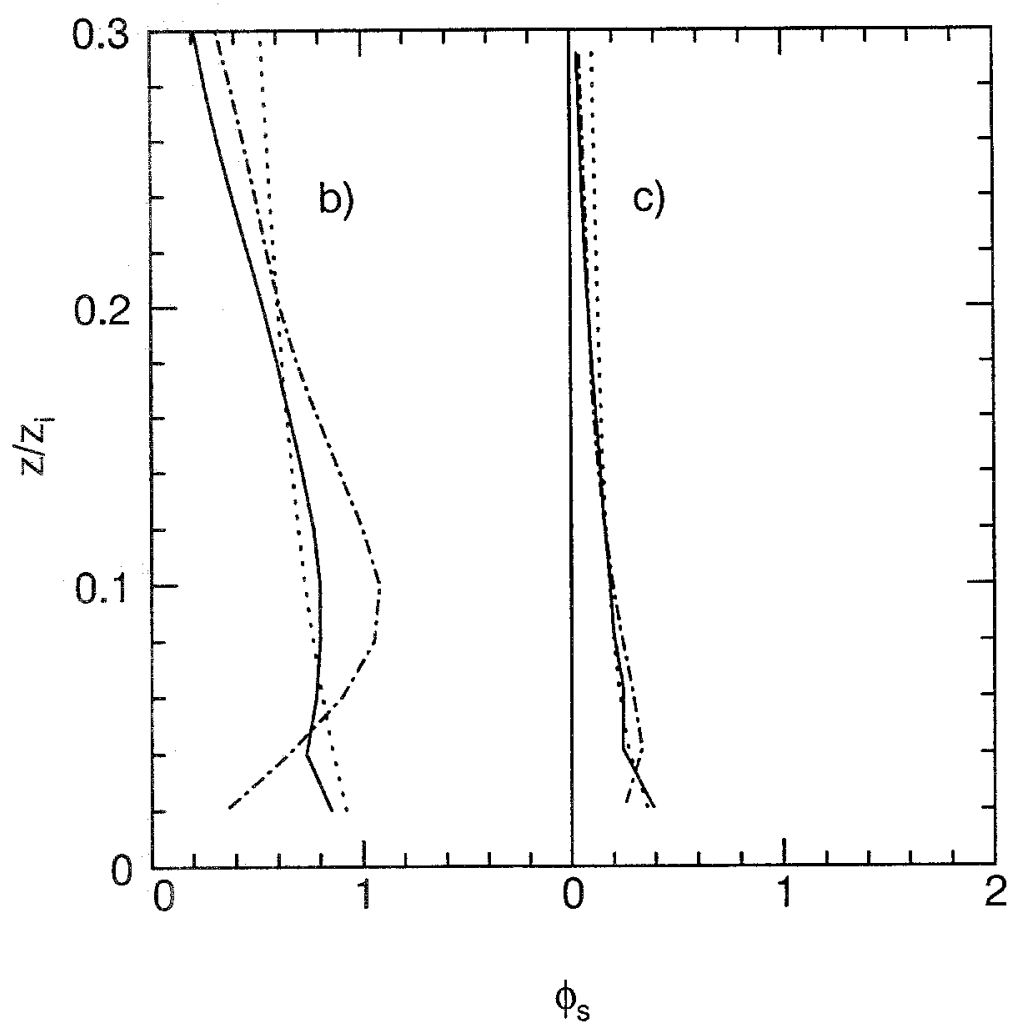

Fig. 4. Potential temperature stability function profiles; dotted line similarity theory, dashed-dotted line baseline model, solid line new model; for the same flows as in Figure 1.

The stability function $\phi_{s}$ and potential temperature $\theta$, shown in Figures 4 and 5 , possess the same general features as their velocity field counterparts with the new SGS model. With the new model, close agreement between the computed $\phi_{s}$ and the similarity prediction is observed up to about $z=0.2 z_{i}$, while a distinct logarithmic region is found in the potential temperature profile for simulations $S B 3$ and $B$. Note that the derivative of the potential temperature at the first grid point is in close agreement to similarity theory even though no attempt is made to match these quantities explicitly. The results from the baseline model deviate sharply from similarity theory for simulation $S B 3$.

\subsection{GRID-ANISOTROPY EFFECTS}

In order to study the influence of grid anisotropy and to some extent grid resolution, on the present results, a grid sensitivity experiment was carried out where the vertical resolution was held constant while the horizontal spacing was gradually refined. Simulations of a strong shear-weakly convective PBL with effective mesh aspect ratios $\Delta x / \Delta z=6.0,4.7,3.0$ and 1.5 were carried out, viz., $S B 1, S B 2, S B 3$ and $S B 4$. This set of simulations provides a clue as to 


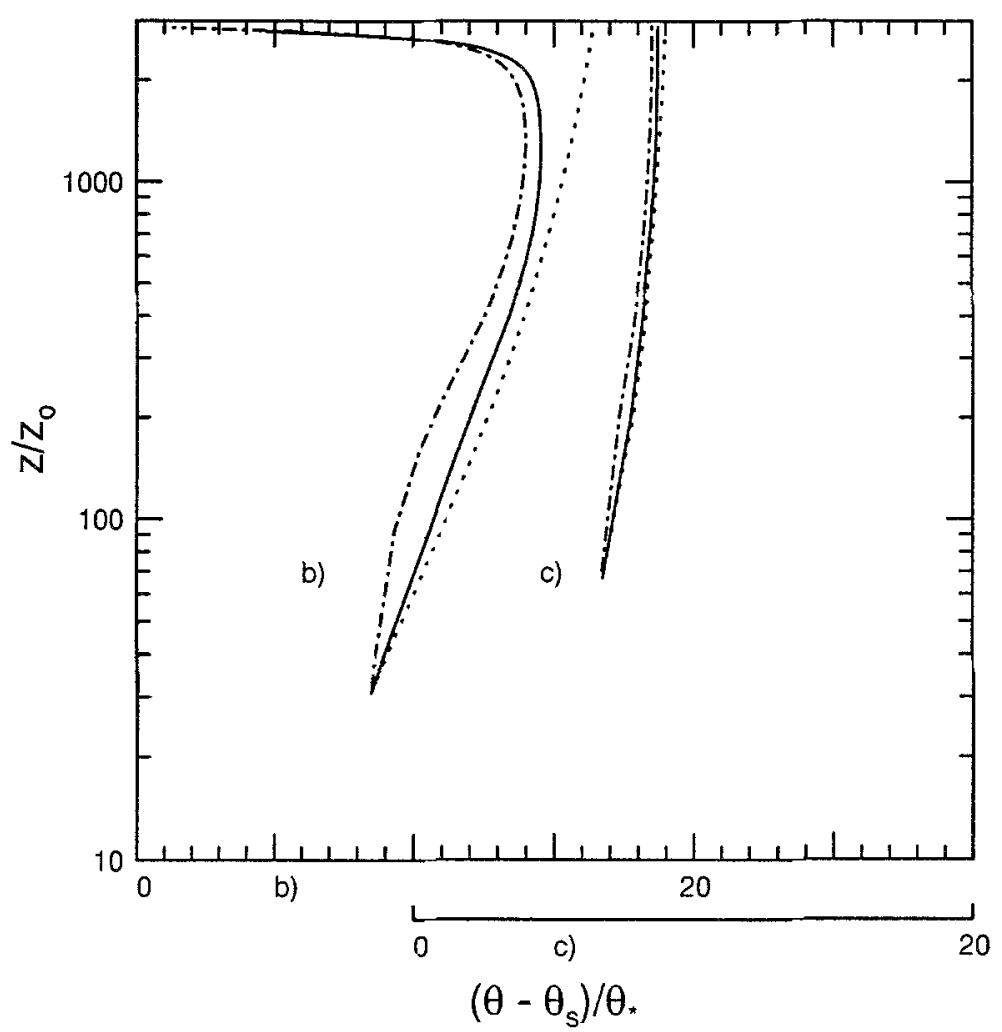

Fig. 5. Potential temperature profiles; dotted line similarity theory, dashed-dotted line baseline model, solid line new model; for same flows as in Figure 1.

whether the deviation from similarity theory using the baseline model can be corrected with enhanced grid resolution or with a more isotropic grid spacing. At the same time, the effects of grid resolution using the new model can also be studied.

Computed estimates of the stability functions, mean wind and mean potential temperature profiles are compared to similarity theory predictions in Figures 6 . 7,8 and 9. Examination of the variations reveals, as the horizontal grid resolution becomes finer, that a substantial error still exists using the baseline model, although a closer inspection of $\phi_{m}$ suggests that the error occurs nearer the surface as $\Delta x / \Delta z \rightarrow 1$. With the new SGS model, an extended logarithmic region is observed in both the wind speed and potential temperature profiles shown in Figures 7 and 9, respectively. The extent of the region over which the new SGS model and similarity theory agree extends up to $\approx 100 \mathrm{~m}$ or $\approx 0.2 z_{i}$, which is expected considering that $-z_{i} / L<1$. On the basis of the velocity and temperature profiles, the extent of the logarithmic region is about 5 to 6 times greater with the new SGS model compared to results with the baseline model. Improve- 


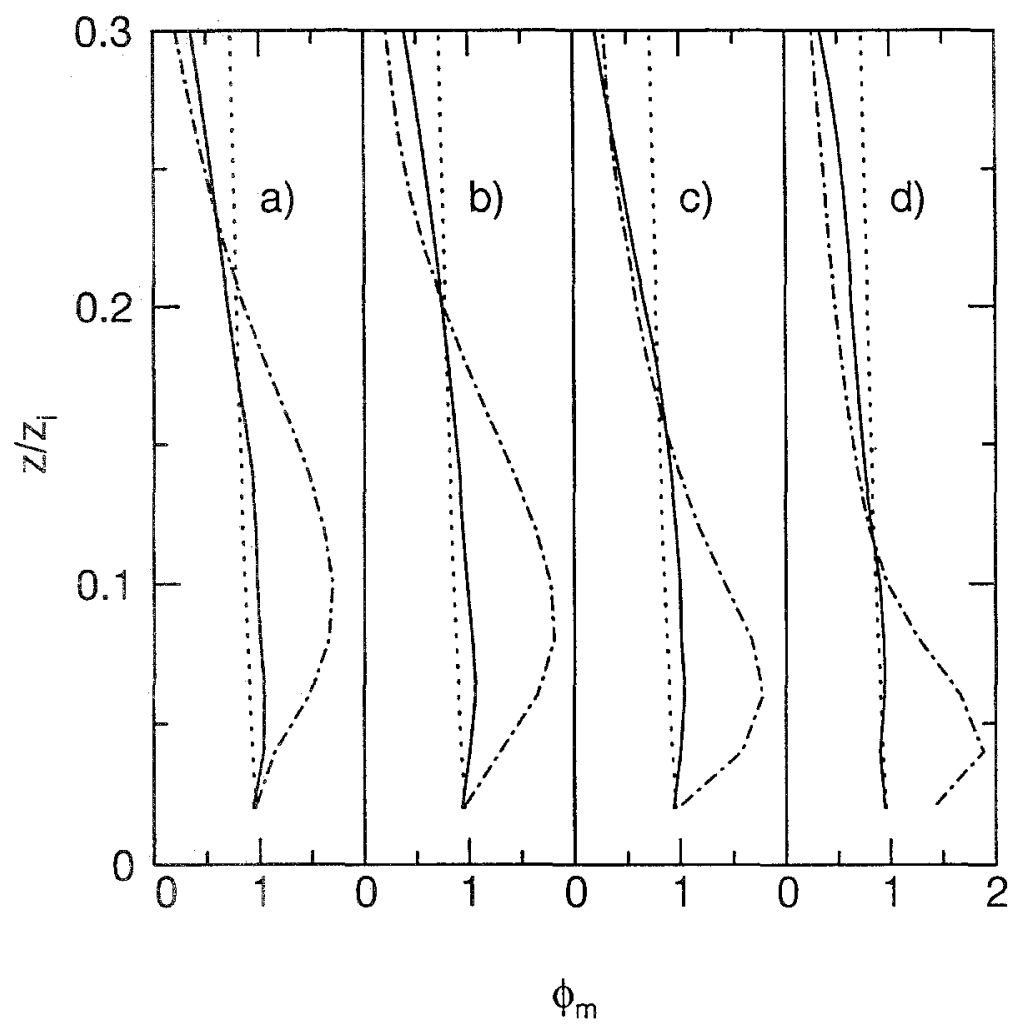

Fig. 6. Momentum stability function profiles as a function of grid resolution weakly convective-strong shear flow simulation, dotted line similarity theory, dashed-dotted line baseline model, solid new line model; (a) grid $50 \times 50 \times 75$, (b) grid $64 \times 64 \times 75$, (c) grid $100 \times 100 \times 75$, (d) grid $192 \times 192 \times 75$.

ments in the wind and temperature profiles are found at all grid resolutions used. Although no explicit correction is applied to the SGS model for heat flux, noticeable improvements are found in the stability function $\phi_{s}$ and potential temperature profiles $\theta$. This suggests that errors in prior simulations were primarily confined to the treatment of the SGS velocity fields for flows with strong shear and small buoyancy effects. The small variation in the computed mean profiles for cases $S B 1, S B 2, S B 3$ and $S B 4$ with the new SGS model implies that the manner in which the mean-field eddy viscosity is computed properly takes into account variations due to grid resolution. Some explorations where the vertical resolution was changed (not shown) indicate that the new model produces mean profiles which are also independent of the vertical mesh spacing.

The variation of the isotropy factor with grid resolution is shown in Figure 10. As anticipated previously, the region over which $\gamma$ alters the isotropic eddy viscosity is reduced with increasing grid resolution. This is due to the resolved small-scale strains which increase with finer resolution. From Equation (29), 


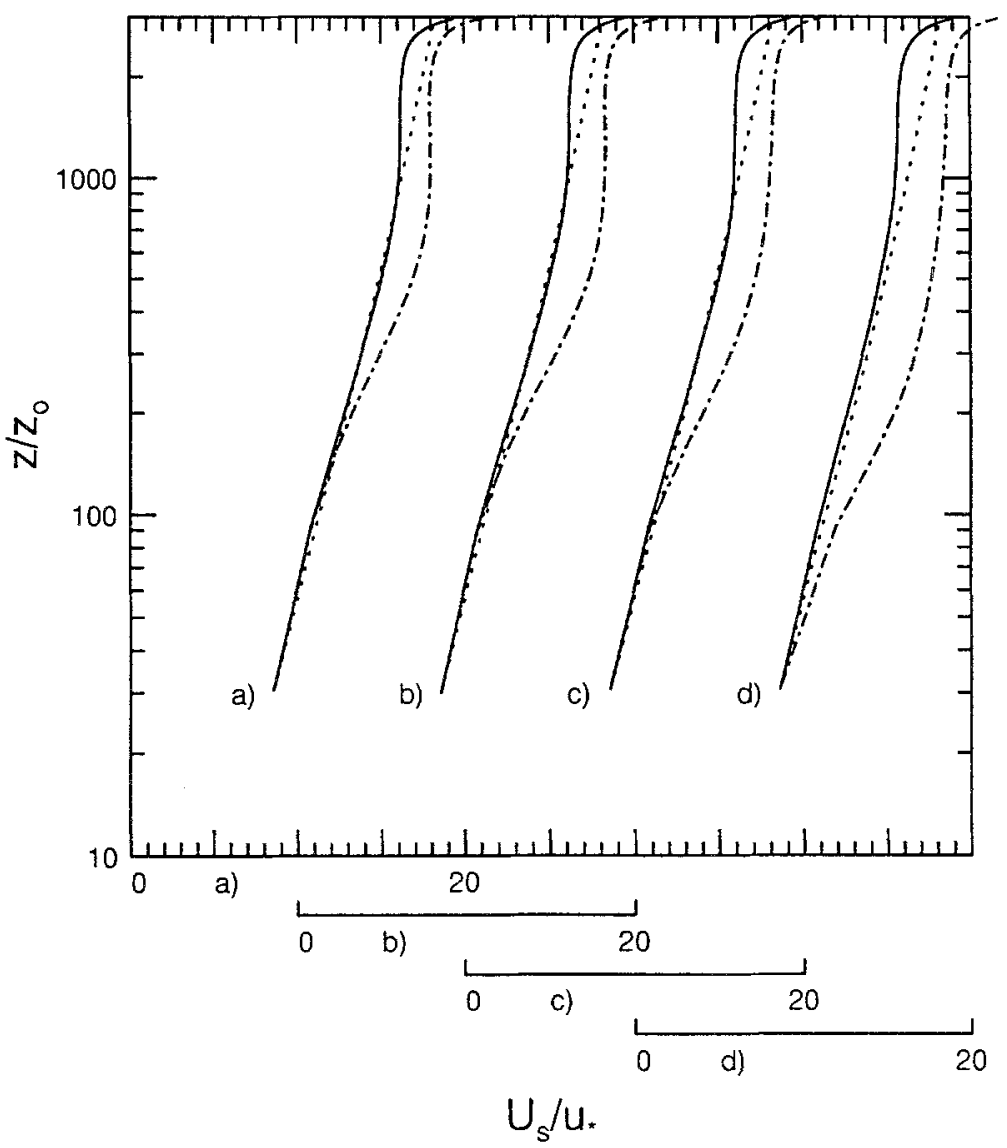

Fig. 7. Wind speed profiles for the same conditions as in Figure 6.

the equivalent Smagorinsky constant near the surface is $\tilde{C}_{s} \approx 0.12$ to 0.13 depending on the grid resolution, which is close to the optimum value suggested by Deardorff (1970) for channel flows.

\subsection{Second-MOMEnt statistics}

The changes to the mean wind and temperature profiles introduced by the new SGS model are also reflected in the second-moment turbulent statistics. However, since the largest changes are observed in the shear-dominated neutral flow, this simulation will be the only one shown here.

Inspection of Table II shows that the surface stress (i.e., $u_{*}^{2}$ ) increases by about $15 \%$ with the new model compared to the baseline computation. This increase largely results from the mean-field eddy viscosity, which dissipates mean kinetic energy.

The vertical variation of the total turbulent kinetic energy, resolved plus SGS velocity variances, and vertical momentum flux normalized by $u_{*}^{2}$ are shown 


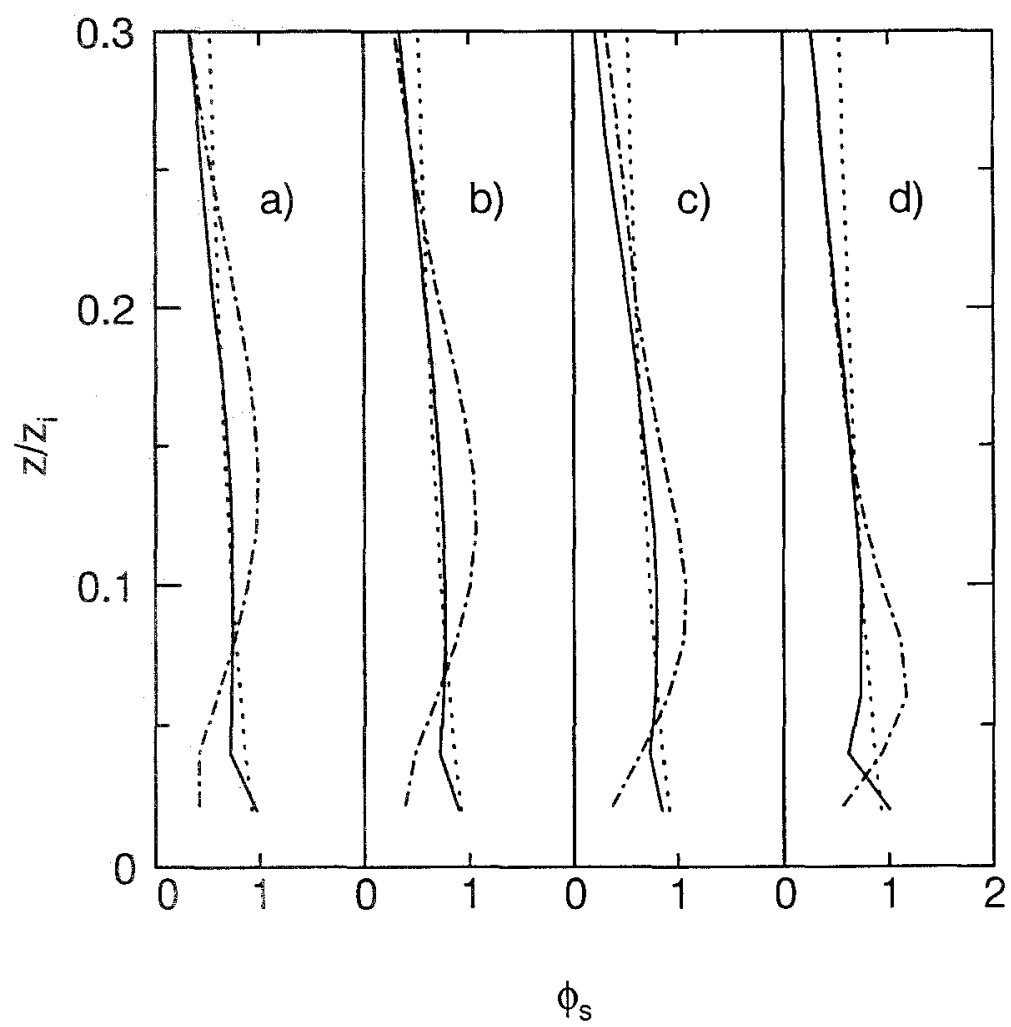

Fig. 8. Potential temperature stability function profiles for same conditions as in Figure 6.

in Figures 11, 12 and 13, respectively. Above the surface layer the shape of the normalized kinetic energy profile with the new SGS model is similar to the baseline model. In the surface layer, the SGS contribution to the total kinetic energy is reduced and more energy lies in the resolved motions with the new SGS model. This is a consequence of the reduced eddy viscosity in the nearwall region and the change in shape in the mean velocity profile. Notice that the normalized peak kinetic energy with the new model is about $5 \%$ smaller than the baseline result, and occurs at a slightly lower height. In Figure 12, the maximum $\left\langle u^{2}\right\rangle$ variance with the new SGS model is approximately $4.7 u_{*}^{2}$ whereas with the baseline model, the peak is about $8 \%$ larger, $\left\langle u^{2}\right\rangle=5.0 u_{*}^{2}$. At the same time, the $\left\langle v^{2}\right\rangle$ and $\left\langle w^{2}\right\rangle$ variances, obtained with the new model, are increased in the surface layer compared to the baseline results.

The change in shape in the $\left\langle w^{2}\right\rangle$ profile in the surface layer with the new SGS model is especially apparent. Near the boundary, the local minimum and sharp increase in the $\left\langle w^{2}\right\rangle$ variance obtained with the baseline model is replaced by a smooth increase followed by a gradual decrease with the new model. It should be noted that Mason and Thomson (1992) obtained a vertical $\left\langle w^{2}\right\rangle$ profile similar to our baseline model after the introduction of stochastic backscatter. They interpret 


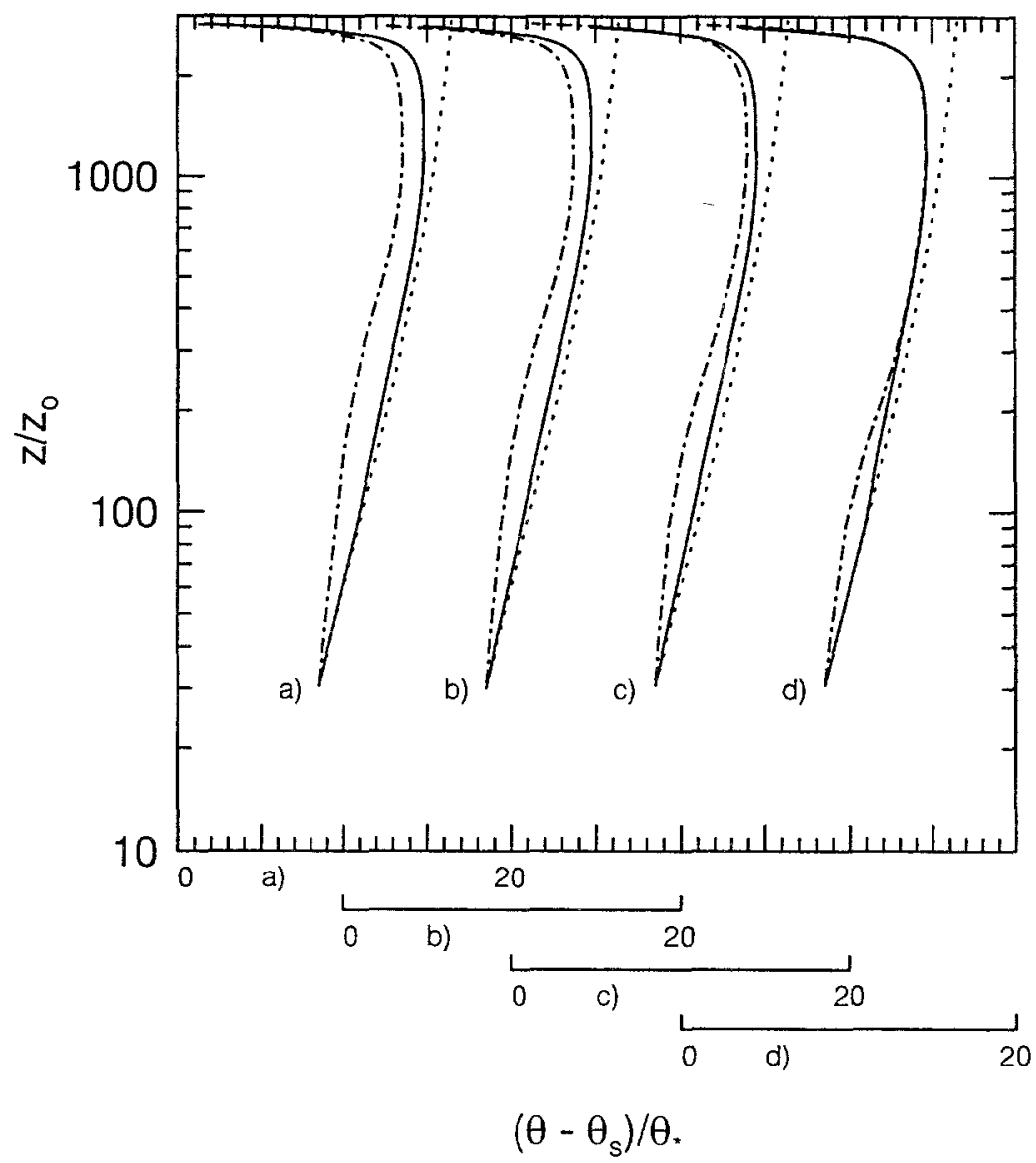

Fig. 9. Potential temperature profiles for same conditions as in Figure 6.

this as a result of using isotropic estimates for their SGS variances. Mason and Thomson (1992) applied further corrections to their estimates of the SGS $\left\langle w^{2}\right\rangle$ variance, and then obtained a $\left\langle w^{2}\right\rangle$ profile similar to that found with our new SGS model. Meanwhile, our maximum $\left\langle u^{2}\right\rangle$ and $\left\langle v^{2}\right\rangle$ variances with the new model are almost identical to the values computed by Mason and Thomson (1992) using their backscatter model.

The flux profiles shown in Figure 13 also show that the resolved motions are enhanced by the reduced eddy viscosity below $z / z_{i}=0.1$ with the new SGS model. Inspection of the profiles indicates that the vertical location where the resolved and SGS contributions are equal occurs at a smaller $z$ with the new model as compared to the baseline model. (Note that the flux profile $\langle u w\rangle / u_{*}^{2}$ at the surface is slightly less than unity because of a small but noticeable contribution from the spanwise flux $\langle v w\rangle$. The latter arises because of the presence of Coriolis rotation, e.g., Coleman et al. (1990). 


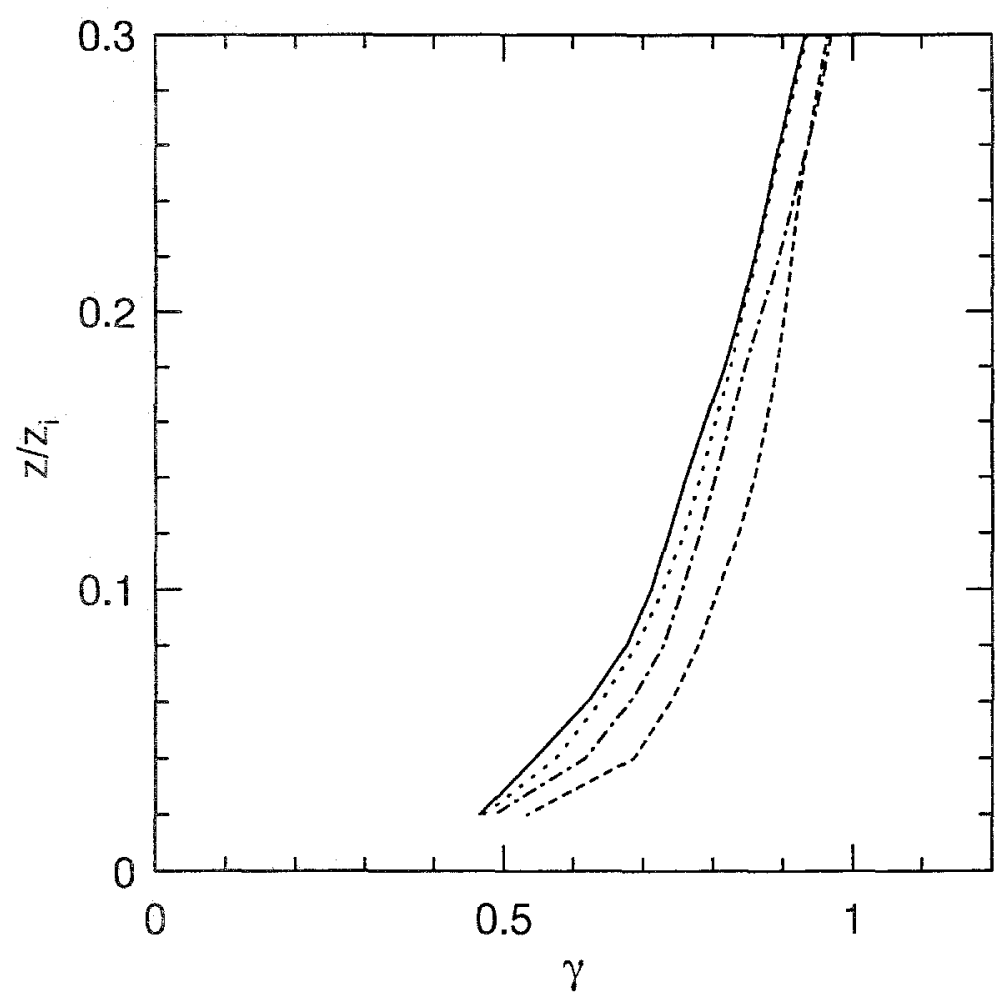

Fig. 10. Isotropy factor for different grid resolutions of weakly convective-strong shear flow; solid line grid $50 \times 50 \times 75$, dotted line grid $64 \times 64 \times 75$, dashed-dotted line grid $100 \times 100$ $\times 75$, dashed line grid $192 \times 192 \times 75$.
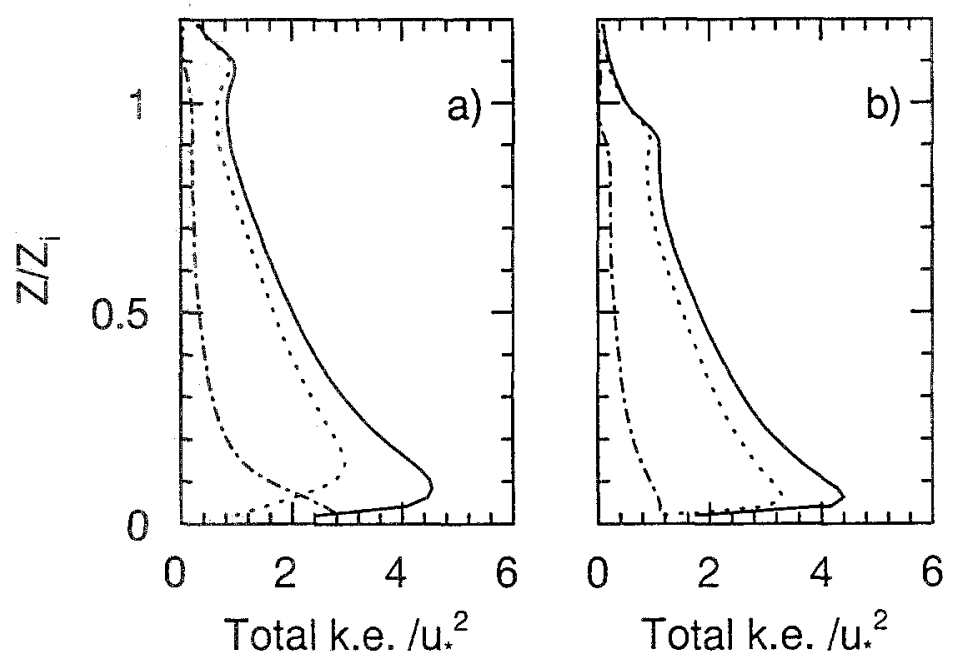

Fig. 11. Kinetic energy profile neutral flow simulation; solid line total, dotted line resolved, dashed-dotted line subgrid scale; (a) baseline model, (b) new model. 

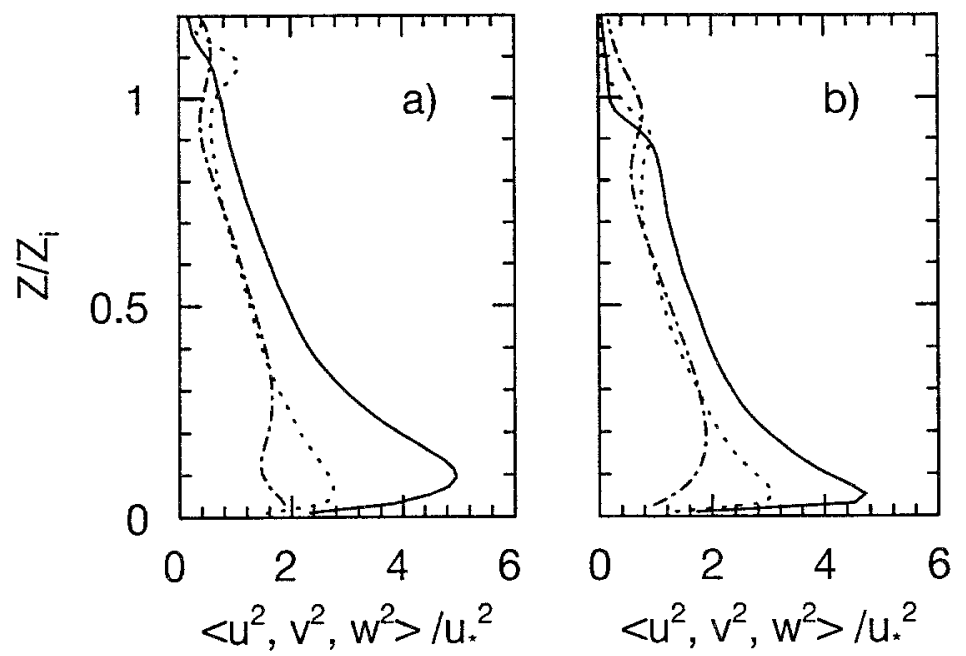

Fig. 12. Resolved plus SGS velocity variance profiles neutral flow simulation; solid line $\left\langle u^{2}\right\rangle$, dotted line $\left\langle v^{2}\right\rangle$, dashed-dotted line $\left\langle w^{2}\right\rangle$; (a) baseline model, (b) new model.
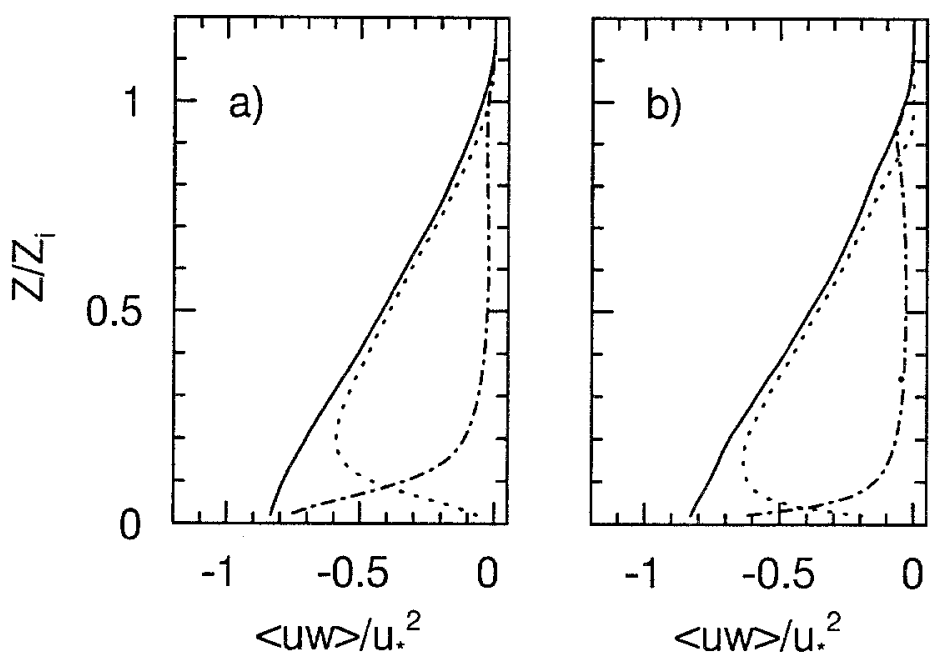

Fig. 13. Momentum flux profile neutral flow simulation; $\langle u w\rangle$ solid line total, dotted line resolved, dashed-dotted line subgrid scale; (a) baseline model, (b) new model.

\subsection{Flow Visualization}

The effects of the new model on the detailed flow structures can be illustrated by comparing flow patterns obtained with the baseline and new models. In Figure 14 , snapshots of the $u, w$, and $u w$ flow fields in the $x-y$ plane at a single vertical location well within the surface layer, $z=0.06 z_{i}$, at nearly the same instant in time for simulation $S$ are shown. The shading and contouring scheme 

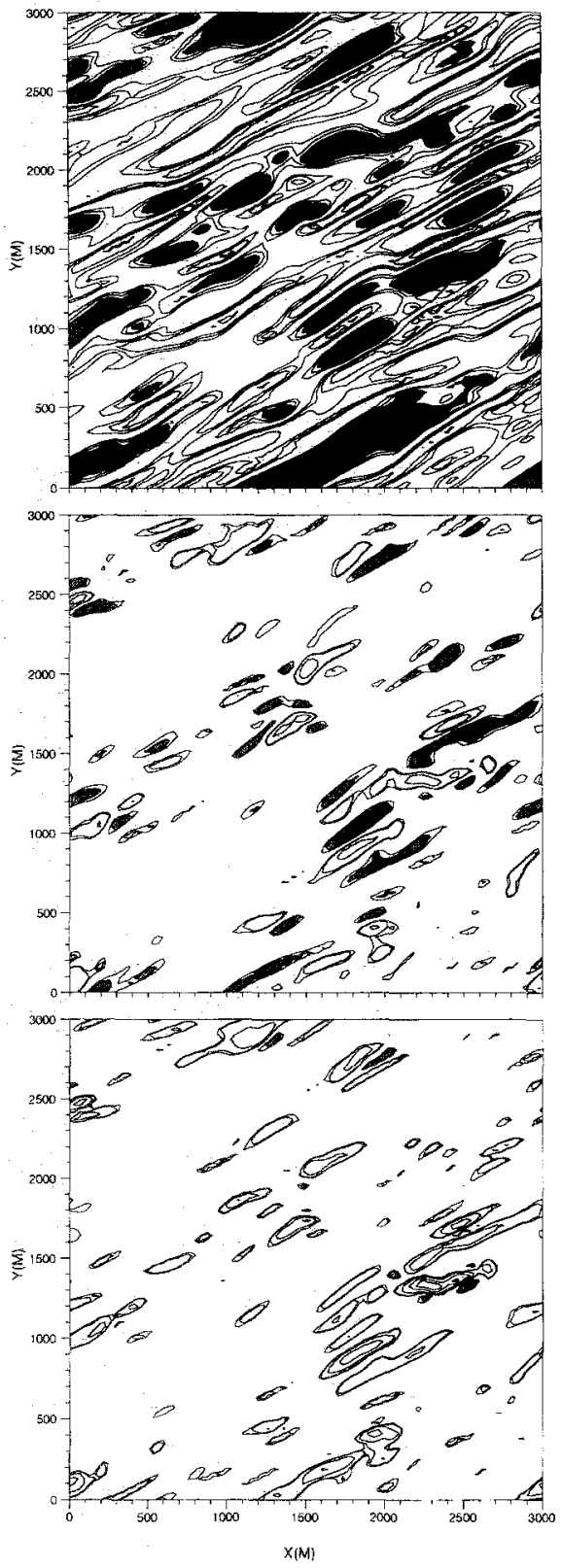
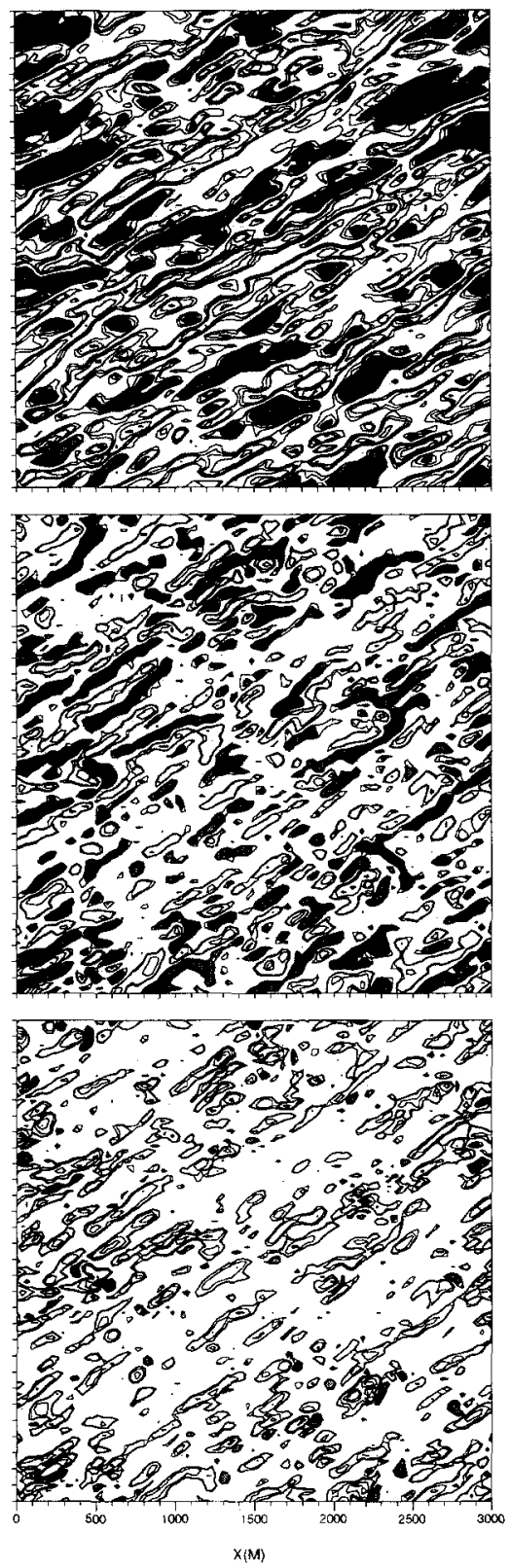

Fig. 14. Contours with baseline (left side) and new (right side) SGS models; top $u$ field, middle $w$ field, and bottom $v w$ field. $u$ contours $(-3,-2,-1.5,-1,-0.75,0.75,1,1.5,2,3)$, dark (light) shading values larger (smaller) than $0.75(-0.75): w$ and $u w$ contours $(-1.5,-1,-0.5,-0.25$, $-0.2,0.2,0.25,0.5,1,1.5$ ) dark (light) shading values larger (smaller) than $0.25(-0.25)$.

is selected to highlight the areas of maximum and minimum intensities in each field. 
Inspection of the figures reveals that the new model preserves the same flow structures as the baseline model, but with significantly more detail. For instance, the alignment of the streaky structures, readily observed in the $u$ field, is nearly identical with the baseline and new SGS models. At the same time, the regions where positive (negative) $w$ fluctuations occur are aligned with negative (positive) $u$ fluctuations. This alignment is also clearly shown in the $u w$ flux contours, which are predominantly negative. These features are discussed in more detail in Moeng and Sullivan (1994). With the new model, however, finer details appear in all flow patterns. This is a result of the smaller SGS eddy viscosity with the new SGS model which is less dissipative relative to the resolved motions. The appearance of more energetic higher frequency motions with the new SGS model is consistent with the stochastic backscatter results of Mason and Thomson (1992); however, the source of these high frequency resolved motions differs. In the present case, the high frequency motions arise because of a reduced SGS eddy diffusivity, whereas in Mason and Thomson (1992), random stochastic forcing at the SGS level raises the energy content of the resolved motions. We cannot state with certainty that the results from the new model shown in Figure 14 are a better representation of actual flow fields than given by the baseline model owing to the lack of such detailed measurements. However, since the new model has a lower eddy viscosity and larger, more dominant, resolved motions compared to the baseline model results, we feel that the results from the new model are in all likelihood a better representation of the actual flow.

\subsection{Model SENSITIVITY}

By trial and error, we found that the shape of the velocity profiles and their derivatives are controlled by the two new features in our SGS eddy viscosity model, viz., $\nu_{T}$ and $\gamma$. Solutions obtained with $\nu_{T}$ alone and the baseline model for $\nu_{t}$ were too dissipative in the near-wall region. This is expected since the effective Smagorinsky constant for the baseline model is $C_{s}=0.18$, while prior channel flow simulations have used $C_{s}=0.1$ (e.g., Deardorff, 1970). Thus a reduction in the SGS eddy viscosity $\nu_{t}$ in the near-wall region is required. However, reducing $\nu_{t}$ by $\gamma$ in the absence of $\nu_{T}$, although beneficial, resulted in excessive mean-velocity gradients compared to similarity theory predictions somewhere near the surface. Thus we conclude that for our infinite Reynolds number PBL simulations, both a mean-field eddy viscosity and a reduction of the fluctuating eddy viscosity are required in order to achieve agreement with similarity theory. We also found that if one chooses the constant $C_{K} L_{m}$ in the mean-field eddy viscosity for one flow at a single grid resolution, the selected constant was not necessarily optimum for use in other flows or grid resolutions. The dynamical procedure adopted by Equation (23) attempts to account for variations in $\nu_{T}$ caused by changes in grid resolution. It should be noted that additional improvements in the wind speed profiles may perhaps be achieved with further refinements to the isotropy factor. If one were to reduce $\gamma$ more in 
the near-wall region, then $\nu_{T}$ would increase, although not linearly, according to Equation (23). This in turn would provide additional leverage over the shape of the mean profile. The present model, however, has been shown to be reasonably successful with various shear and buoyancy combinations ranging from neutral to strongly convective even with modest grid resolutions.

\section{Conclusions}

In the surface layer where SGS motions are comparable to (or even dominate) the resolved motions, a LES for the PBL is sensitive to the type of SGS model. In order to investigate this sensitivity, a series of LES was calculated for PBLs which vary from neutrally stratified to strongly convective flows. In addition, a grid-anisotropy sensitivity study was carried out with horizontal-to-vertical grid aspect ratios of $6.0,4.7,3.0$ and 1.5 .

We find that the turbulent-kinetic-energy eddy-viscosity model suggested by Deardorff (1980) and used by Moeng (1984) produces deviations from MoninObukhov similarity forms near a bounding surface. The deviation from similarity forms (or error) is readily observed in the wind and temperature profiles, and to a greater extent in their dimensionless vertical derivatives (i.e., the stability functions). Overshoot by a factor of two in the stability function is found for our neutral PBL simulation, similar to that found previously by Mason and Thomson (1992). However, with substantial buoyant forcing, the error is negligible.

In order to improve the simulations in the near-wall region, a two-part eddyviscosity model is proposed. The new model adds to the turbulent-kinetic-energy formulation for the eddy viscosity used by Moeng (1984) a contribution from the mean shear. The mean-field eddy viscosity, unlike the prior models of Schumann (1975) and Grotzbach and Schumann (1977), is adjusted along with the flow evolution so that the mean shear matches similarity theory at the first computational grid point above the surface. Although the mean-field eddy viscosity is only important near the wall, it leads to a substantial improvement in the profiles of wind speed and potential temperature throughout the whole surface layer. In addition, an isotropy factor $\gamma$, proportional to the ratio of the resolved fluctuating strain and the horizontal mean strain, accounts for the anisotropy in the SGS motions near the wall. This factor reduces the fluctuating eddy viscosity by more than $50 \%$ in the near-wall region. The equivalent Smagorinsky constant near the surface is then about 0.12 , which is close to the empirical values used for previous channel-flow simulations.

The results of our simulations with the new model clearly show logarithmic regions in both the wind speed and potential temperature profiles for all cases examined. Similar improvements are observed in the profiles of the stability functions for momentum and temperature. Agreement with similarity forms is found up to about $0.1-0.2 z_{i}$. These results are also found to be independent of grid anisotropy. A comparison of the second-moment turbulent statistics shows 
that the resolved motions contain more energy and the surface stress is about $15 \%$ greater with the new model. Also, $\left\langle u^{2}\right\rangle$ with the new model is slightly reduced very near the boundary. Inspection of the instantaneous flow patterns reveals that the same fundamental flow structures are present independent of the SGS model; with the new model, however, finer detail appears because of lower SGS eddy viscosity. Additional advantages of the new SGS model are that it is simple to implement and requires little additional computational cost.

\section{Acknowledgements}

We thank Keith Ayotte and Jeff Weil for their help in the preparation and review of this document. Part of this work is supported by ONR Government Order N00014-92-F-0117. Also, some of the computations were performed through the courtesy of the Cray Computer Corporation.

\section{References}

Bardina, J., Ferziger, J. H., and Reynolds, W. C.: 1983, Improved Turbulence Models Based on Large Eddy Simulation of Homogeneous, Incompressible, Turbulent Flows. Thermosciences Report TF-19. Stanford University.

Businger, J. A., Wyngaard, J. C., Izumi, Y., and Bradley, E. F.: 1971, 'Flux-Profile Relationships in the Atmospheric Surface Layer', J. Atmos. Sci. 28, 181-189.

Cebecci, T. and Bradshaw, P.: 1988, Physical and Computational Aspects of Convective Heat Transfer. Springer-Verlag, $187 \mathrm{pp}$.

Canuto, V. M. and Minotti, F.: 1993, 'Stratified Turbulence in the Atmosphere and Oceans: A New Subgrid Model', J. Atmos. Sci. 50, 1925-1935.

Clark, R. A., Ferziger, J. H., and Reynolds, W. C.: 1979, 'Evaluation of Subgrid-Scale Models Using an Accurately Simulated Turbulent Flow', J. Fluid Mech. 91, 1-16.

Coleman, G. N., Ferziger, J. H., and Spalart, P. R.: 1990, 'A Numerical Study of the Turbulent Ekman Layer', J. Fluid Mech. 213, 313-348.

Deardorff, J. W.: 1970, "Numerical Simulation of Turbulent Channel Flow at Large Reynolds Number', J. Fluid Mech. 41, 452-480.

Deardorff, J. W.: 1980, 'Stratocumulus-Capped Mixed Layers Derived from a Three-Dimensional Model', Boundary-Layer Meteorol. 18, 495-527.

Germano, M., Piomelli, U., Moin, P. and Cabot, W. H.: 1991: 'A Dynamic Subgrid-Scale Eddy Viscosity Model', Phys. Fluids A 7, 1760-1771.

Gerz, T. and Schumann, U.: 1989, 'Direct Simulation of Homogeneous Turbulence and Gravity Waves in Sheared and Unsheared Stratified Flows', in Durst et al. (eds.), Turbulent Shear Flows, Vol. 7, Springer-Verlag, Berlin, 27 pp.

Grotzbach, G. and Schumann, U.: 1977, Direct Numerical Simulation of Turbulent Velocity-, Pressure-, and Temperature-Fields in Channel Flows. Symposium on Turbulent Shear Flows, The Pennsylvania State University, April 18-20.

Horiuti, K.: 1993, 'A Proper Velocity Scale for Modeling Subgrid-Scale Eddy Viscosities in Large Eddy Simulation', Phys. Fluids 5, 146-157.

Mahrt, L. and Gibson, W.: 1992, 'Flux Decomposition into Coherent Structures', Boundary-Layer Meteorol. 60, 143-168.

Mason, P. J. and Callen, N. S.: 1986, 'On the Magnitude of the Subgrid-Scale Eddy Coefficient in Large-Eddy Simulations of Turbulent Channel Flow', J. Fluid Mech. 162, 439-462.

Mason, P. J. and Thomson, D. J.: 1992, 'Stochastic Backscatter in Large-Eddy Simulations of Boundary Layers', J. Fluid Mech. 242, 51-78. 
Moeng, C.-H.: 1984, "A Large-Eddy-Simulation Model for the Study of Planetary Boundary-Layer Turbulence", J. Atmos. Sci. 41, 2052-2062.

Moeng, C.-H. and Wyngaard, J. C.: 1988, 'Spectral Analysis of Large-Eddy Simulations of the Convective Boundary Layer', J. Atmos. Sci. 45, 3575-3587.

Moeng, $\mathrm{C}_{n}-\mathrm{H}$. and Sullivan, P. P.: 1994, 'A Comparison of Shear and Buoyancy Driven PlanetaryBoundary Flows', J. Atmos. Sci. 51, 999-1022.

Moin, P. and Kim, J.: 1982, 'Numerical Investigation of Turbulent Channel Flow', J. Fluid Mech. $118,341-377$.

Nieuwstadt, F. T. M. and Brost, R. A.: 1986, 'The Decay of Convective Turbulence', J. Atmos. Sei. 43, 532-546.

Nieuwstadt, F. T. M., Mason, P. J., Moeng, C.-H., and Schumann, U.: 1993, 'Large-Eddy Simulation of the Convective Boundary Layer: A Comparison of Four Computer Codes', in Durst et al. (eds.), Turbulent Shear Flows, Vol. 8, Springer-Verlag, Berlin, 431 pp.

Piomelli, U., Moin, P., and Ferziger, J. E.: 1988, 'Model Consistency in Large Eddy Simulation of Turbulent Channel Flows', Phys. Fluids 31, 1884-1891.

Piomelli, U., Ferziger, J. H., and Moin, P.: 1989, 'New Approximate Boundary Conditions for Large Eddy Simulations of Wall Bounded Flows', Phys. Fluids A 6, 1061-1068.

Reynolds, W. C.: 1989, 'The Potential and Limitations of Direct and Large Eddy Simulations', Whither Turbulence? or Turbulence at the Crossroads, Cornell University.

Rogallo, R. S. and Moin, P.: 1984, 'Numerical Simulation of Turbulent Flows', Ann. Rev. Fluid Mech. 16, 99-137.

Schmidt, H. and Schumann, U.: 1989, 'Coherent Structure of the Convective Boundary Layer Derived from Large-Eddy Simulations', J. Fluid Mech. 200, 511-562.

Schumann, U:: 1975, 'Subgrid Scale Model for Finite Difference Simulations of Turbulent Flows in Plane Channels and Annuli', J. Comp. Phys. 18, 376-404.

Schumann, U., Grotzbach, G.; and Kleiser, L.: 1980, 'Direct Numerical Simulation of Turbulence', Prediction Methods for Turbulent Flows, Editor Wolfgang Kollmann.

Schumann, U.: 1991, 'Subgrid Length-Scales for Large-Eddy Simulation of Stratified Turbulence', Theoretical and Computational Fluid Dynamic 2, 279-290.

Schumann, U.: 1993, 'Stochastic Backscatter of Turbulence Energy and Scalar Variance from Random Subgrid-Scale Fluxes', Phys. Fluids, Preprint.

Scotti, A., Meneveau, C., and Lilly, D. K.: 1993, 'Generalized Smagorinsky Model for Anisotropic Grids', Phys. Fluids 5, 2306-2308.

Smagorinsky, J.: 1963, 'General Circulation Experiments with the Primitive Equations. I. The Basic Experiment', Mon. Weather Rev. 91, 99-164.

Spalart, P. R.: 1988, 'Direct Simulation of a Turbulent Boundary Layer up to $R_{\theta}=1410$ ', J. Fluid Mech. 187, 61-98.

Tennekes, H. and Lumley, J. L.: 1972, A First Course in Turbulence, The MIT Press, 266 pp.

Van Driest, E. R.: 1956. 'On the Turbulent Flow near a Wall', J. Aero. Sci. 23, 1007-1011.

Wyngaard, J. C.: 1984, Large-Eddy Simulation: Guidelines for its Application to Planetary Boundary Layer Research, US Army Research Office Contract No. 0804.

Wyngaard, J. C.: 1988, 'Structure of the PBL', in A. Venkatram and J. C. Wyngaard (eds.), Lectures on Air Pollution Modeling, AMS, Boston, pp. 9-61.

Yakhot, A., Orszag, S., Yakhot, V., and Israeli, M.: 1989, 'Renormalization Group Formulation of Large-Eddy Simulations', J. Sci. Comp. 4, 139-158.

Yoshizawa, A.: 1989, 'Subgrid-Scale Modeling with a Variable Length Scale', Phys. Fluids A 1, 1293-1295. 